\title{
Transverse electron cooling of heavy molecular ions
}

\author{
C. Krantz $\odot,{ }^{1, *}$ H. Buhr, ${ }^{1,2}$ M. Grieser, ${ }^{1}$ M. Lestinsky $\odot,{ }^{1}$ O. Novotný $\odot,{ }^{1,3}$ S. Novotny $\odot,{ }^{1}$ \\ D. A. Orlov, ${ }^{1}$ R. Repnow, ${ }^{1}$ A. S. Terekhov, ${ }^{4}$ P. Wilhelm, ${ }^{1}$ and A. Wolf ${ }^{1}$ \\ ${ }^{1}$ Max-Planck-Institut für Kernphysik, 69117 Heidelberg, Germany \\ ${ }^{2}$ Faculty of Physics, Weizmann Institute of Science, Rehovot 76100, Israel \\ ${ }^{3}$ Columbia Astrophysics Laboratory, Columbia University, New York, New York 10027, USA \\ ${ }^{4}$ Institute of Semiconductor Physics, 630090 Novosibirsk, Russia
}

(Received 10 January 2021; accepted 29 March 2021; published 3 May 2021)

\begin{abstract}
Transverse electron cooling of heavy molecular ions has been studied at the Test Storage Ring (TSR). Electron beams from a cold GaAs:(Cs,O) photocathode, with kinetic energies down to $31 \mathrm{eV}$, have been used for cooling of singly-charged ions of masses up to $41 \mathrm{u}$. We believe that these are the heaviest singly-charged ions for which successful electron cooling has been reported so far. Transverse ion-beam emittances $\ll 1 \mu \mathrm{m}$ were reached after typically several seconds of cooling time. The measured transverse cooling rates agree with a simple binary-collision model, assuming a transverse electron temperature of approximately $1 \mathrm{meV} / k_{\mathrm{B}}$. The results serve as benchmark for electron cooling at the new Cryogenic Storage Ring, which uses the same photocathode electron source and is targeting singly-charged ions of even higher mass.
\end{abstract}

DOI: 10.1103/PhysRevAccelBeams.24.050101

\section{INTRODUCTION}

Electron coolers were originally developed to enhance the brilliance of proton beams in storage rings or synchrotrons [1]. As was realized early, their merged-beams geometry also enables their usage as high-resolution electron targets for stored ions. Once beam cooling is complete, careful de-tuning of the electron velocity allows one to study electron-ion collisions at energies down to the $\mathrm{meV}$ scale [2,3], as are of interest in low-temperature plasmas. Naturally, the latter are characterized by relatively low equilibrium ion charge states, and, with the advent of suitable heavy-ion storage rings, electron cooling has been applied to ions of ever-lower charge-to-mass ratio $q / m_{\mathrm{i}}$. The cooling rate is approximately proportional to $q^{2} / m_{\mathrm{i}}$. Thus, even heavy ions can be cooled efficiently as long as their charge states $q$ are sufficiently high [4-6]. Singly or weakly charged ions can be challenging, as their mass outweighs the effect of charge, so that cooling rates become small compared to protons.

\footnotetext{
*orresponding author.

claude.krantz@mpi-hd.mpg.de,c.krantz@gsi.de.

Present address: GSI Helmholtz Centre for Heavy Ion Research, 64291 Darmstadt, Germany.

Published by the American Physical Society under the terms of the Creative Commons Attribution 4.0 International license. Further distribution of this work must maintain attribution to the author(s) and the published article's title, journal citation, and DOI. Open access publication funded by the Max Planck Society.
}

Motivated by precision studies of electron recombination [7-16], electron cooling has been applied to many singlycharged molecular cations at the Test Storage Ring (TSR) [17] of the Max Planck Institute for Nuclear Physics (MPIK), whose high-resolution Electron Target [18] was complemented by a cold photocathode electron source in the process [19-21]. In this paper, we review some of these experiments under aspects of accelerator physics. They involved electron-cooled, coasting beams of singly-charged molecules with masses up to $41 \mathrm{u}$. Because of the low stored ion currents, observation of the longitudinal cooling process based on pickup signal analysis was not possible. However, the transverse cooling rates and equilibrium beam properties could be measured via single-particle imaging of recombination products. To the extent of our knowledge, these are the heaviest singly-charged ions for which electron cooling has been studied.

While electron collision experiments with similarly massive and even heavier molecular ions have been realized at CRYRING, they did not rely on electron cooling as a means to reduce phase-space spreads of the beams, specifically because the expected cooling times were longer than the storage lifetime of the ions as limited by residualgas collisions [22-24]. The latter can lead to relatively fast beam loss, given the low velocities at which singly-charged heavy ions must be stored. Our work at the TSR demonstrated that electron cooling can successfully prepare low-emittance beams for experiments even under such conditions. In that sense, it is an important step toward electron cooling at the Cryogenic Storage Ring (CSR), 
recently commissioned at MPIK as a replacement for the TSR [25]. The CSR is an electrostatic ion storage and cooler ring targeting even heavier molecular ions $\left(q / m_{\mathrm{i}} \geq 1 / 160 \mathrm{u}\right)$ at $300 \mathrm{kV}$ electric rigidity [26]. The CSR electron cooler re-uses the cold photocathode electron source from TSR [27], a key element of the work discussed here. A first CSR experiment on electron recombination of a (relatively light) molecular ion has been published recently [28].

The structure of this paper is as follows: Section II provides an overview of the experimental methods. It presents the TSR, the high-resolution photocathode Electron Target, as well as its associated product particle detector systems that served as ion-beam diagnostics. These descriptions are kept short, as all systems have been presented at length in previous publications. Section III presents a basic formalism of transverse electron cooling as needed to interpret the measurements. In Sec. IV, the individual experiments are presented, analyzed using the developed methods, and put into context by comparison to theoretical expectations. Section V closes with a summary and outlook to future electron cooling activities at MPIK.

\section{METHODS}

Until end of 2012, the heavy-ion facility of MPIK consisted of three main accelerators: a 12-MV Tandem Van-de-Graaff accelerator, a smaller 3-MV single-stage Van-de-Graaff Pelletron, and an RFQ/drift-tube-linac combination. The pre-accelerators were followed by a common optional post-accelerator structure [29]. All machines could inject beam into the 1.4-Tm heavy-ion storage ring TSR [17].

Figure 1 provides an overview of the layout of TSR. The inset displays the horizontal and vertical beta functions $\beta_{x}$ and $\beta_{y}$ as well as the horizontal dispersion $D_{x}$ for the standard operation mode of the ring [30].

The TSR vacuum system reached pressures of a few $10^{-11}$ mbar all along its circumference of $55.42 \mathrm{~m}$ [30]. Storage times of several tens of seconds were possible, even for heavy molecular ions.

\section{A. The TSR "Electron Target"}

While one electron cooler (5 in Fig. 1) had already been part of the original design of TSR [31], a second device, the Electron Target, was later added to the ring (2 in Fig. 1). Even though its name does not reflect that function, the "Target" was in fact an electron cooler optimized for lowest beam temperatures (cf. Fig. 2). Its beam transport system, described in detail by Sprenger et al. [18], featured a superconducting gun solenoid for strong expansion of the electron beam, followed by a long, adiabatic acceleration section. For low electron velocities, as in the experiments discussed here, the full acceleration voltage was applied already in the electron gun, so that expansion took place at

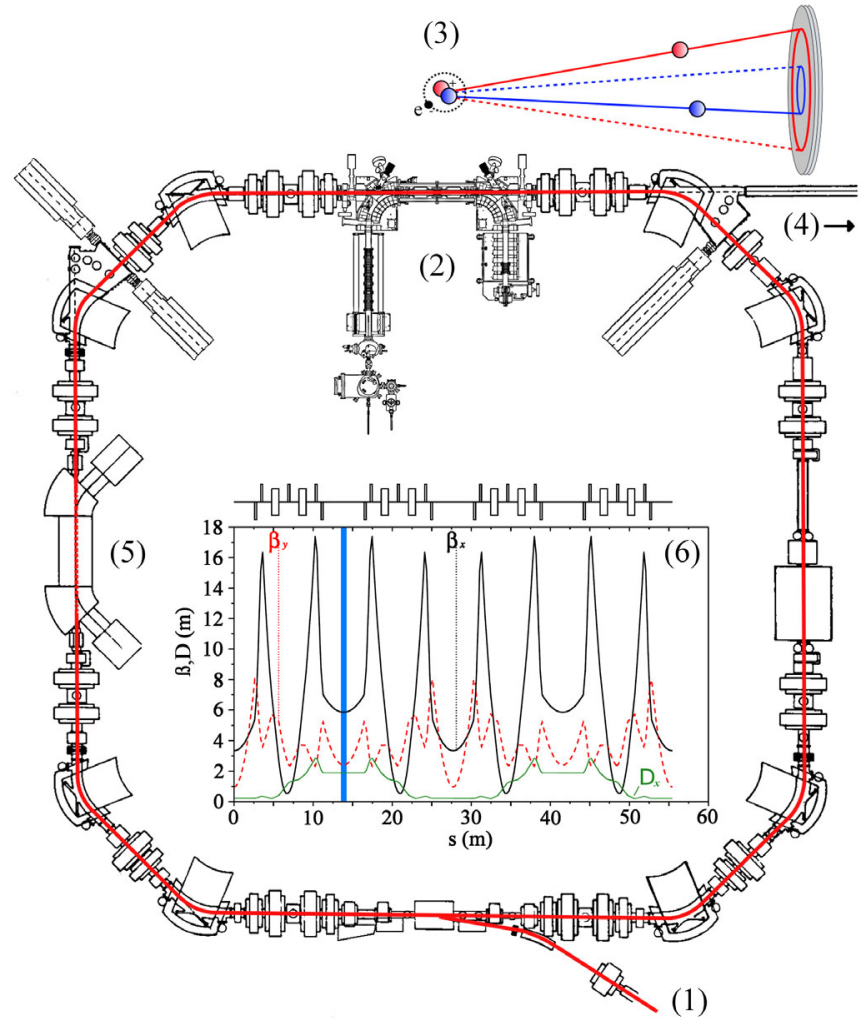

FIG. 1. Overview of the heavy-ion storage ring TSR: After injection from a linear accelerator (1, not shown), singly-charged molecular ions were electron-cooled in the photocathode Electron Target (2). Recombination (3) led to neutral products that left the ring via an extraction beam line and were recorded by a fragmentimaging detector (4, not shown). The primary electron cooler of TSR (5) is shown only schematically, as it is not discussed here. The inset (6) shows the beta $\left(\beta_{x}\right.$ and $\left.\beta_{y}\right)$ and horizontal dispersion $\left(D_{x}\right)$ functions of the TSR in standard operation mode [30]. The solid blue line in the graph indicates the position of the Electron Target.

the final beam energy. $90^{\circ}$ toroidal coils merged and separated the electron and ion beams. The interaction solenoid had a length of $1.5 \mathrm{~m}$. The region where the noncollinearity of the beams is negligible with respect to the transverse electron temperature defines the "effective length" $l_{\text {eff }}$ of the cooler. For the experiments discussed here, we expect $l_{\text {eff }}=1.30(5) \mathrm{m}$.

After interaction with the ions, the electron beam was decelerated and dumped into an analyzer Faraday cup. Via a movable pin-hole aperture, the latter was able to measure the transverse beam profile, which allowed us to determine the electron density $n_{e}$ in the cooler beam [18].

Besides a conventional thermionic emitter, the Target could be equipped with a cryogenic photocathode electron source $[19,20]$. The photocathode setup is the only major subsystem of the TSR that is reused at the new CSR facility $[25,26]$. It is based on GaAs: $(\mathrm{Cs}, \mathrm{O})$ cathodes, prepared to the state of negative electron affinity [32]. Transmissionmode illumination of the photocathode, in our case by an 


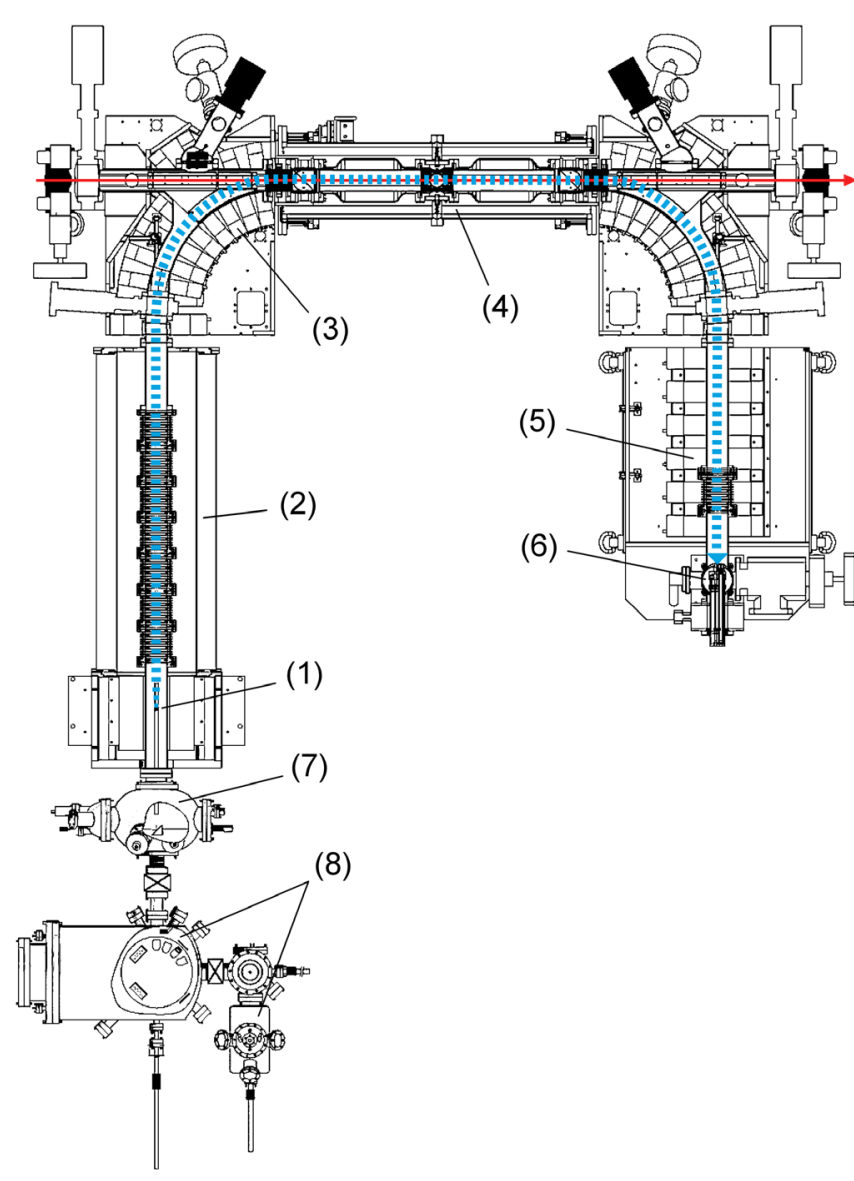

FIG. 2. Overview of the Electron Target (2 in Fig. 1). The electron beam (blue dashed line) was emitted from a cold GaAs: $(\mathrm{Cs}, \mathrm{O})$ photocathode (1) embedded in a strong axial magnetic field. Magnetic expansion of the beam took place at the entrance of the adiabatic acceleration section (2). For low ion velocities, as discussed here, the latter was not used, as electrons reached their final energy already in the electron gun. A toroidal coil (3) merged the electrons with the ion beam (solid red line). After a $1.5-\mathrm{m}-$ long interaction section (4) the beams were separated by another toroid. After deceleration (5), the electrons were dumped into a Faraday cup (6). The GaAs:(Cs,O) emitter was illuminated in transmission mode by an $808-\mathrm{nm}$ laser from the photocathode setup (7). The latter contains facilities for in-vacuum cleaning and reactivation of cathodes (8), allowing quasicontinuous operation.

808-nm laser, combined with space-charge-limited extraction, allows formation of electron beams having a temperature close to that of the GaAs bulk. Cooled by liquid nitrogen, the latter can be an order of magnitude colder than a thermionic emitter [33]. Technically, operation of the GaAs:(Cs, O) cathodes is significantly more involved, as the useful lifetime of a prepared cathode sample is limited by degradation of the $(\mathrm{Cs}, \mathrm{O})$ activation layer, which can proceed quickly at elevated DC emission current. For fast ion beams, this sets a practical limit to the current at $\sim 1 \mathrm{~mA}$, which allows a GaAs sample to operate for $24 \mathrm{~h}$ [21]. In electron cooling of slow ions, as in the experiments described here, the maximum current is space-chargelimited to lower values already by the small acceleration voltage in the electron gun, and several days of operation become possible with no compromise on beam density. The setup contains facilities for in-vacuum replacement, cleaning and reactivation of a number of cathodes, and thus allows quasicontinuous operation of the electron gun. The long-term behavior of the GaAs photocathodes has been discussed in a previous publication [34].

Combined with the photocathode, the Electron Target reached very high energy resolution in electron-ion collision experiments [35]. For the reasons discussed in Sec. III, its much lower electron temperature compared to the original TSR electron cooler was of great advantage regarding cooling of singly-charged heavy ions [36]. Thus, in TSR experiments involving heavy molecular ions, electron cooling was soon performed using the photocathode Electron Target exclusively.

\section{B. Neutral-product detectors}

The Target was followed by an extraction beam line for neutral products, as typically result from electronic recombination of singly-charged molecular ions. Two imaging-capable, single-particle detector systems were available [37].

The first was an 8-cm diameter MCP-phosphor-screen detector, located at a distance of $12.2 \mathrm{~m}$ from the center of the electron cooler. CCD imaging of the screen allowed to determine the impact positions of individual recombination products on the MCP. In addition, the detector was equipped with a highly-precise ( $\leq 1 \mathrm{~ns})$ optical timing system, that could measure arrival time differences of several neutral fragments emerging from a single recombination event [38]. Although the MCP is not sensitive to the kinetic energies of individual particles, fragment identification is possible via a statistical procedure as described by Strasser et al. [39].

The second detector was a $10 \times 10 \mathrm{~cm}^{2}$ surface-barrier diode equipped with a $128 \times 128$ multi-strip readout [40]. It could alternatively be moved into the extraction beam line at a distance of $9.6 \mathrm{~m}$ from the Target center. Its timing system was much less precise, so that only transverse (2D) fragment momentum imaging was possible. However, its detection principle allowed direct identification of molecular fragments via their massdependent energy deposition.

While both detector systems were experimental systems, primarily designed for studies of molecular recombination processes in the cooler beam, their ability to reconstruct the phase-space distribution of the recombining ions also made them powerful beam diagnostics, as explained below.

\section{Beam diagnostics}

Because of the relatively low saturation limits of the neutral-product imaging detectors, TSR experiments on 
molecular ions usually involved very low ion beam currents. Typically $10^{4}$ to $10^{6}$ particles were injected at the beginning of each TSR cycle. In combination with the low revolution frequencies (typically $\sim 100 \mathrm{kHz}$, see later Table II) this led to stored ion currents $\lesssim 10 \mathrm{nA}$ and a very weak Schottky noise of the coasting beam. Although the latter was detectable for various molecular beams, and could help with coarse velocity-matching of the electron cooler [41], observation of the (longitudinal) electron cooling process based on Schottky analysis was normally not possible because of the very low signal level.

Fine-tuning of Electron Target settings therefore relied on observation of the transverse cooling process. While the TSR was equipped with a residual-gas-ionization beamprofile monitor [30], its dark count rate was usually too high to detect the weak molecular ion beams. Instead, the recombination signal from the neutral-product imaging detectors (cf. Sec. II B) was used, taking advantage of their much higher signal-to-background ratio.

Compared to the total momentum of a stored ion, the momentum transfer related to recombination in an electron cooler is negligible. Hence the product particle maintains the velocity and direction of its parent ion. For protons, imaging of hydrogen atoms from recombination has been used as diagnostics for the transverse ion beam properties since the early years of electron coolers $[1,42,43]$.

At the TSR, this technique has been extended to singlycharged molecular ions [44]. A complication lies in the fact that molecular ions dominantly recombine via dissociative recombination (DR), which leads to two or more neutral fragments for each recombination event [45].

Because of the significant kinetic-energy release associated with many DR processes, the fragments recede from each other and scatter on the detector at typical distances of a few $\mathrm{cm}$ from the original ion beam axis. Unlike for protons, the distribution of detector hits can thus not be directly interpreted as an image of the extrapolated ion beam envelope. However, the center-of-mass (c.m.) of all fragments from a given recombination event does propagate along the original axis of the parent ion. If the neutral masses can be assigned to the individual detector hits, their c.m. distribution can thus be reconstructed and evaluated analogously to the proton case.

The center of the Electron Target coincides with a beam waist in the TSR (at $s_{0} \simeq 13.9 \mathrm{~m}$ in the coordinate system of Fig. 1). We denote by indices $x$ and $y$ the horizontal and vertical directions transverse to the beam trajectory, equivalent, respectively, to the radial and axial degrees of freedom of ion motion in the TSR. In the standard operation mode of TSR [30], the horizontal and vertical beta function amplitudes at $s_{0}$ are $\beta_{x}=6.0(3) \mathrm{m}$ and $\beta_{y}=2.3(1) \mathrm{m}$, respectively (cf. Fig. 1). From there, the beta functions $\tilde{\beta}_{x, y}(s)$ of the neutral product beam follow the evolution for a drift space. At a distance $d$ from the Target center, we have

$$
\tilde{\beta}_{x, y}\left(s_{0}+d\right)=\beta_{x, y}\left(1+\frac{d^{2}}{\beta_{x, y}^{2}}\right) .
$$

With the horizontal and vertical beam emittances denoted by $\varepsilon_{x}$ and $\varepsilon_{y}$, the sizes of the neutral beam envelopes are

$$
\tilde{\sigma}_{x, y}(s)=\sqrt{\tilde{\beta}_{x, y}(s) \varepsilon_{x, y}} .
$$

For better readability, we introduce the shorthands $\sigma_{x, y}=$ $\tilde{\sigma}_{x, y}\left(s_{0}\right)$ and $\hat{\sigma}_{x, y}=\tilde{\sigma}_{x, y}\left(s_{0}+d\right)$. Equations (1) and (2) can thus be used to calculate the horizontal and transverse envelopes $\sigma_{x}$ and $\sigma_{y}$ of the ion beam in the Electron Target from those of the neutral-product beam $\hat{\sigma}_{x}$ and $\hat{\sigma}_{y}$, measured using a detector at distance $d$ :

$$
\sigma_{x, y}=\hat{\sigma}_{x, y}\left(1+\frac{d^{2}}{\beta_{x, y}^{2}}\right)^{-1 / 2} .
$$

As the Target center $\left(s_{0}\right)$ is a beam waist, the following simple relations between envelope sizes $\sigma_{x, y}$, transverse emittances $\varepsilon_{x, y}$, and beam divergences $\sigma_{x, y}^{\prime}$ hold:

$$
\varepsilon_{x, y}=\frac{\sigma_{x, y}^{2}}{\beta_{x, y}} \quad \text { and } \quad \sigma_{x, y}^{\prime}=\frac{\sigma_{x, y}}{\beta_{x, y}} .
$$

In the following analysis, we define $\hat{\sigma}_{x, y}$ to be the standard deviations of the product c.m. distributions at the detectors. Then also $\sigma_{x, y}$, obtained from Eq. (3), are standard deviations and $\varepsilon_{x, y}$ from Eq. (4) are $68 \%$ transverse beam emittances. In the later Sec. IV we tabulate more commonly used $95 \%$ horizontal and vertical beam emittances defined by $\varepsilon_{x, y}^{95 \%}=4 \varepsilon_{x, y}$.

One should note that, in Eq. (2), we have assumed that the beam envelope at $s_{0}$ is defined only by betatron motion of the ions. In principle, the relative longitudinal momentum spread $\delta p / p$ can additionally contribute to the overall horizontal beam size $\sigma_{x}^{\text {tot }}$ via the nonvanishing horizontal dispersion at the Electron Target $\left(D_{x} \simeq 2 \mathrm{~m}\right.$, cf. Fig. 1), i.e.,

$$
\sigma_{x}^{\mathrm{tot}}=\sqrt{\sigma_{x}^{2}+\left(D_{x} \frac{\delta p}{p}\right)^{2}} \text { with } \quad \sigma_{x}^{2}=\beta_{x} \varepsilon_{x}
$$

This effect can however be neglected: as the longitudinal cooling force is usually greater than the transverse one, the relative momentum spread $\delta p / p$ is expected to be smaller than the divergence $\sigma_{x}^{\prime}=\sigma_{x} / \beta_{x}$. Using $0<\delta p / p<\sigma_{x} / \beta_{x}$ with Eq. (5) yields $\sigma_{x}<\sigma_{x}^{\text {tot }}<\sigma_{x} \sqrt{1+\left(D_{x} / \beta_{x}\right)^{2}}$. With $\beta_{x} \simeq 6.0 \mathrm{~m}$ and $D_{x} \simeq 2.0 \mathrm{~m}$, we thus have $\sigma_{x}<\sigma_{x}^{\text {tot }} \lesssim$ $1.05 \sigma_{x}$. As will become clear in Sec. IV, this effect is too small to be resolved in the experiment. As implicitly stated in Eq. (2) we have, effectively, $\sigma_{x}^{\text {tot }} \approx \sigma_{x}$. 


\section{TRANSVERSE ELECTRON COOLING}

Transverse electron cooling leads to damping of betatron oscillations in the storage ring. We regard the radial $(x)$ and axial $(y)$ betatron motions as independent. Along both directions, the transverse cooling forces $F_{x}$ and $F_{y}$ are functions of the respective ion velocity components $u_{x}$ and $u_{y}$ perpendicular to the electron beam axis. For better readability, we mostly omit the indices $x$ and $y$ while remembering that the formalism applies independently to both degrees of freedom.

\section{A. Basic model}

Traditionally, the cooling force is derived in the binary collision approximation [46], analogously to Bethe stopping of ions in an isotropic electron medium. This leads to the following expression for $F(u)$ [4]

$$
F(u)=m_{\mathrm{i}} \dot{u} \approx-6 \pi\left(\frac{q e^{2}}{4 \pi \epsilon_{0}}\right)^{2} \frac{n_{e} L_{C}}{m_{e}} \frac{u}{|u|^{3}+2 \Delta_{e}^{3}} .
$$

Therein, $q$ is the charge state of the ion, $e$ the elementary charge, and $\epsilon_{0}$ the vacuum permittivity. $m_{e}$ and $m_{\mathrm{i}}$ denote the electron and ion masses, respectively, and $n_{e}$ the electron density. $\Delta_{e}$ is the transverse electron velocity spread, discussed below. The Coulomb logarithm $L_{C}=$ $\ln \left(b_{\max } / b_{\min }\right)$ results from integration over all relevant impact parameters $b$ in the ion-electron scattering process.

Equation (6) neglects the axial magnetic field used to guide the electron beam in most coolers. A thorough theoretical treatment of electron cooling including a finite magnetic field is difficult, as it gives rise to nonlinear dynamics in the ion-electron interaction [47].

Thus, extensions of the original (nonmagnetic) analytic models of electron cooling have been developed in order to account for the field in approximate ways [48]. They predict an overall enhancement of the cooling effect due to the electrons being tightly confined to the field lines, so that their transverse velocity spreads become irrelevant for ion interactions slower than the cyclotron period. These "magnetized" models have gained some experimental support, mainly from measurements of the longitudinal cooling force at various machines [49,50]. However, also quite large discrepancies have been found in some cases, and semiempirical formulas remain in widespread use $[6,51,52]$.

For our transverse cooling experiments, we consistently found that magnetized models predicted much higher cooling rates than observed in reality. In our comparison to theoretical predictions, we thus adopt a nonmagnetic binary collision model as in Eq. (6). Effects of the anisotropic velocity distribution will be discussed in Sec. III C.

\section{B. "Deep" electron cooling}

For large betatron amplitudes, the transverse velocities of the ions can be greater than those of the electrons. Using $|u|^{3} \gg \Delta_{e}^{3}$ in Eq. (6) leads to $|F(u)| \sim u^{-2}$. Analogous to stopping in matter, the force is very weak for fast ions and increases nonlinearly as the particles slow down.

While, for the slow ion beams discussed here, values $|u|>\Delta_{e}$ are reached only for extreme emittances, an additional complication in our experiments is related to the finite electron beam diameter (of 12.5(7) $\mathrm{mm}$ in most cases): for ions performing excursions greater than the electron beam radius, the cooling force vanishes for a certain range of betatron phases, as ions do not interact with the electron beam at every revolution. Transverse damping rates during the early stages of electron cooling therefore strongly depend on the initial emittance of the ion beam, and are difficult to compare among different experiments.

The situation clears up once betatron motion has been sufficiently damped for the ion envelope to become completely embedded in the electron beam. From this moment on, $|u|^{3} \ll \Delta_{e}^{3}$ and Eq. (6) simplifies to

$$
F(u) \approx-3 \pi\left(\frac{q e^{2}}{4 \pi \epsilon_{0}}\right)^{2} \frac{n_{e} L_{C}}{m_{e}} \frac{u}{\Delta_{e}^{3}}=:-2 \lambda m_{\mathrm{i}} u .
$$

This defines the betatron damping rate $\lambda$ for "deep" electron cooling. Over many revolutions of an ion the friction force $F(u)$ (proportional to $-u$ ) leads to exponential amplitude decrease of the betatron oscillation according to $|u(t)| \sim \exp (-\lambda t)$.

In contrast to the initial stages of cooling, the deep cooling rate $\lambda$ is constant, depending only on wellcontrolled experimental parameters.

\section{Transverse cooling rates}

Contrary to the assumption underlying Eq. (6), the velocity distribution of the cooler electrons is usually not isotropic, as the short beam transit time and the axial magnetic guiding field of most coolers suppress coupling between the transverse degrees of electron motion (indices $x$ and $y$ ) and the longitudinal one (index $z$ ). Under the assumption that the velocity spreads mostly stem from thermal electron energy, the electron beam can be characterized by its independent transverse and longitudinal temperatures.

In our nonmagnetic approximation, electron cooling of betatron motion is mostly defined by the transverse velocity spread $\Delta_{e}$. However, the longitudinal spread does have an indirect influence resulting from integration over the 3-dimensional velocity distribution [53]. While kinematic compression in the accelerated beam initially suppresses the longitudinal spread with respect to the transverse, subsequent plasma relaxation leads to a final value that is difficult to predict precisely [3,54-56]. We will consider two limiting cases: a fully isotropic electron velocity distribution, and a "flattened" distribution where the longitudinal velocity spread is so small compared to the transverse that it can be neglected. 
The relation between the transverse velocity spread $\Delta_{e}$ and the corresponding transverse temperature $T_{e}$ of the electron beam is a longstanding source of confusion, as competing definitions are used in the original literature on electron cooling. This has first been pointed out by Bell and Bell [53], and we follow their reasoning.

We write the transverse velocity of an electron with respect to the mean beam axis as $\vec{v}_{\perp}=\vec{v}_{x}+\vec{v}_{y}$. We take $\Delta_{e}$ to be the r.m.s. spread of that velocity in the electron ensemble, i.e., $\Delta_{e}^{2}=\left\langle\left|\vec{v}_{\perp}\right|^{2}\right\rangle$. As electron motion along $x$ and $y$ is coupled by the magnetic guiding field, the corresponding thermal energies are equal: $m_{e}\left\langle\left|\vec{v}_{x}\right|^{2}\right\rangle=$ $m_{e}\left\langle\left|\vec{v}_{y}\right|^{2}\right\rangle=k_{\mathrm{B}} T_{e}$. With the definition of $\vec{v}_{\perp}$ from above we thus have $\Delta_{e}=\left(2 k_{\mathrm{B}} T_{e} / m_{e}\right)^{1 / 2}$.

With this, and for the case of an isotropic velocity distribution (i.e., for $\left\langle\left|\vec{v}_{z}\right|^{2}\right\rangle=\left\langle\left|\vec{v}_{\perp}\right|^{2}\right\rangle / 2=k_{\mathrm{B}} T_{e} / m_{e}$ ), Bell and Bell derive a deep transverse cooling rate [53]

$$
\lambda_{\text {iso }}=\frac{2 \sqrt{2 \pi}}{3}\left(\frac{q e^{2}}{4 \pi \epsilon_{0}}\right)^{2} \frac{n_{e}}{m_{e} m_{\mathrm{i}}} L_{C}\left(\frac{m_{e}}{k_{\mathrm{B}} T_{e}}\right)^{3 / 2},
$$

which agrees with Eq. (7) to within $1 \%$. Notably, this also agrees with the early result obtained by Spitzer [57] for the temperature relaxation rate of an ion-electron plasma, if one again considers the case where the ion velocities are much smaller than those of the electrons. Note that Eq. (8) describes the damping of velocity rather than temperature-slower by a factor 2-and that the presence of betatron oscillation leads to another factor- 2 decrease of the rate compared to free ion motion as discussed by Spitzer [53,57].

For the case of a flattened electron velocity distribution $\left(\left\langle\left|\vec{v}_{z}\right|^{2}\right\rangle \ll\left\langle\left|\vec{v}_{\perp}\right|^{2}\right\rangle / 2\right)$, Bell and Bell obtain [53]

$$
\lambda_{\text {flat }}=\frac{\pi \sqrt{2 \pi}}{2}\left(\frac{q e^{2}}{4 \pi \epsilon_{0}}\right)^{2} \frac{n_{e}}{m_{e} m_{\mathrm{i}}} L_{C}\left(\frac{m_{e}}{k_{\mathrm{B}} T_{e}}\right)^{3 / 2},
$$

i.e., at given $T_{e}$ the betatron damping rate is higher by a factor $\simeq 2.4$ compared to the isotropic case of Eq. (8).

For the Coulomb logarithm we assume, in both cases (8) and (9), a value of

$$
L_{C}=\ln \left(\frac{b_{\max }}{b_{\min }}\right)=\ln \left(4 \pi \sqrt{\frac{2}{n_{e}}} \frac{\left(\epsilon_{0} k_{\mathrm{B}} T_{e}\right)^{3 / 2}}{e^{3} q}\right) .
$$

Therein, the maximum impact parameter $b_{\max }$ is identified with the screening length $r_{D}=\Delta_{e}\left(\epsilon_{0} m_{e} / n_{e} e^{2}\right)^{1 / 2}$ in the transverse plane. $b_{\min }$ is found from the reasoning that, if the ion velocity can be neglected, the maximum momentum transferred in a single collision is equal to $2\left(k_{\mathrm{B}} T_{e} m_{e}\right)^{1 / 2}$ for a given degree of freedom. Because of the logarithmic nature of $L_{C}$, these assumptions have only a minor impact on the model.

\section{Scaling laws}

In the experiment, the stage of deep transverse electron cooling is characterized by the time $\tau_{\text {cool }}$ during which the size of the ion beam envelope shrinks exponentially by a factor $1 / e$. It is the inverse of the cooling rate $\lambda$, scaled by the ratio of storage ring circumference $C$ and electron cooler effective length $l_{\text {eff }}$ :

$$
\tau_{\mathrm{cool}}=\frac{C}{l_{\mathrm{eff}}} \frac{1}{\lambda} .
$$

As mentioned above, for the TSR Electron Target, $C=55.42 \mathrm{~m}$ and $l_{\text {eff }}=1.30(5) \mathrm{m}$, resulting in a scaling factor $C / l_{\text {eff }} \simeq 43$.

Disregarding the logarithmic dependence of $L_{C}$ on $T_{e}$ and $n_{e}, \tau_{\text {cool }}$ is thus expected to scale like

$$
\tau_{\mathrm{cool}} \sim \frac{C m_{\mathrm{i}} T_{e}^{3 / 2}}{l_{\mathrm{eff}} q^{2} n_{e}} .
$$

Equation (12) shows why electron cooling of molecular ions is challenging: On the one hand-in contrast to highly charged atomic ions-molecular ions usually carry a charge $q=1$, as it is often not possible to strip more valence electrons without breaking the molecular bonds. On the other hand, the total ion mass $m_{\mathrm{i}}$ is naturally quite large. These two facts directly lead to long cooling times according to Eq. (12).

In addition, the low charge-to-mass ratio of the ions limits the velocity at which they can be stored, given the maximum rigidity of the ring. As the electron extraction energy must be correspondingly low, the maximum electron cooler current becomes limited by the Child-Langmuir law [58]. Hence, $\tau_{\text {cool }}$ cannot be arbitrarily reduced via an increase of $n_{e}$ for such ion beams. With this, it is clear that a low effective value of $T_{e}$ is imperative for betatron cooling of massive singly-charged ions.

Directly after extraction, the electron temperature is defined by that of the emitting cathode $T_{\text {cath }}$. A cold emitter is therefore of great advantage [27]. Via adiabatic magnetic expansion, $T_{e}$ can be decreased further with respect to $T_{\text {cath }}$ according to

$$
T_{e} \approx \frac{T_{\text {cath }}}{\alpha},
$$

where $\alpha$ is ratio of magnetic fields at the cathode and in the interaction section of the cooler [50,56]. After expansion and acceleration of the beam, the transverse temperature $T_{e}$ is practically not affected by plasma relaxation processes (as opposed to the longitudinal temperature where these effects dominate) [3].

In magnetized models of electron cooling, the effective value of $T_{e}$ is reduced further as cyclotron motion adiabatically averages-out the transverse electron motion for a 
certain class of ion collisions, so that only the (lower) longitudinal temperature remains relevant in those cases [48,51]. Our nonmagnetic binary collision model would thus underestimate the cooling rates if magnetization effects were significant in the experiments. As we show in Sec. IV C, this appears not to be the case.

For fast ( few MeV/nucleon) ion beams, field imperfections of the cooler solenoid or suboptimal alignment of electron and ion beams can mimic an increased transverse temperature $T_{e}$ [59]. For the heavy molecular ions discussed here, these effects are strongly suppressed by the low electron energies and are not believed to have played a role.

\section{E. Equilibrium beam size}

Electron cooling is ultimately limited by competing heating effects acting on the ion beam, which rise in magnitude as the beam envelope shrinks. For the TSR, intrabeam scattering (IBS) is the limiting effect counteracting transverse cooling [17]. Hence, near the end of the cooling process, the beam size $\sigma$ evolves as

$$
\frac{1}{\sigma} \frac{\mathrm{d} \sigma}{\mathrm{d} t}=\lambda_{\mathrm{IBS}}(\sigma)-\frac{1}{\tau_{\mathrm{cool}}},
$$

where $\lambda_{\text {IBS }}$ is the IBS heating rate, itself depending on the beam size. A full theory of IBS for strong-focusing machines has been developed by Martini [60]. For the TSR, Artikova et al. found that $\lambda_{\mathrm{IBS}}$ could be described well by the simplified formula [61]

$$
\lambda_{\mathrm{IBS}} \approx a_{x} \frac{q^{4}}{\left(m_{\mathrm{i}} / \mathrm{u}\right)^{2}}\left(\frac{c}{f_{0} C}\right)^{3} \frac{N_{\mathrm{i}}}{\sigma^{\gamma}},
$$

where $N_{\mathrm{i}}$ is the number of stored ions, $f_{0}$ their revolution frequency in the ring, and $m_{\mathrm{i}} / \mathrm{u}$ the ion mass relative to the atomic mass unit. $a_{x}$ and $\gamma$ are empirical constants. The exponent $\gamma$ is expected to be close to 5 for a coasting beam and is thus responsible for a sharp onset of IBS in the final stage of electron cooling. Artikova et al. obtained values of $a_{x} \simeq 4 \times 10^{-13} \mathrm{~mm}^{\gamma} / \mathrm{s}$ and $\gamma \simeq 4.68$ by measuring the horizontal IBS heating rate of beams of ${ }^{12} \mathrm{C}^{6+}$ at $73.3 \mathrm{MeV}$ in the TSR [61].

From Eq. (14), we expect an IBS-limited equilibrium ion beam size after cooling

$$
\sigma^{\mathrm{f}} \approx\left(a_{x} \frac{q^{4} N_{\mathrm{i}} \tau_{\text {cool }}}{\left(m_{\mathrm{i}} / \mathrm{u}\right)^{2}}\left(\frac{c}{f_{0} C}\right)^{3}\right)^{1 / \gamma} .
$$

While the horizontal and vertical beam sizes $\sigma_{x, y}$ vary along the ring lattice, Eq. (4) relates them to the constant emittances $\varepsilon_{x, y}$. At the center of the Electron Target, the horizontal and vertical beam divergences $\sigma_{x, y}^{\prime}$ correspond to transverse ion velocity spreads

$$
\sigma_{x, y}^{v}=\frac{\sigma_{x, y}}{\beta_{x, y}} f_{0} C .
$$

In equilibrium between electron cooling and IBS heating, $\sigma_{x, y}^{v}$ can be interpreted as thermal velocity spreads, and transverse ion temperatures

$$
T_{\mathrm{i}}^{x, y}=\frac{\sigma_{x, y}^{2} f_{0}^{2} C^{2} m_{\mathrm{i}}}{\beta_{x, y}^{2} k_{\mathrm{B}}}
$$

can be associated to the respective degrees of freedom of the betatron motion.

\section{EXPERIMENT}

Electron cooling has been applied to many molecular ion beams in the TSR. Motivated by questions on molecular quantum dynamics and astrophysics, many of the experiments have already led to specialized publications in these fields [7-16]. The data were normally analyzed in equilibrium conditions, excluding phases of ion beam cooling.

However, in many cases the neutral fragment detectors did record data also during beam preparation. In fact, they were actively used as beam diagnostic devices to help with alignment of electron and ion beams. Here, we revisit some of these experiments with focus on analysis of the transverse cooling process using the methods developed above.

\section{A. Datasets}

In order of rising mass, the ions included in this study are $\mathrm{H}_{3}^{+}[9,11], \mathrm{HF}^{+}$[8], $\mathrm{DF}^{+}$[10], $\mathrm{N}_{2} \mathrm{H}^{+}, \mathrm{DCO}^{+}$[12], $\mathrm{CF}^{+}$ [7], $\mathrm{HS}^{+}$[14], ${ }^{16} \mathrm{O}^{18} \mathrm{O}^{+}, \mathrm{H}^{35} \mathrm{Cl}^{+}$[13], and $\mathrm{D}_{2}{ }^{37} \mathrm{Cl}^{+}$[16]. These datasets have been chosen for their similar operating parameters with regard to the photocathode electron gun of the Target.

Except for the special cases discussed below, the magnetic field at the electron gun was $0.8 \mathrm{~T}$, as opposed to $0.04 \mathrm{~T}$ in the remaining cooler solenoids, equivalent to a magnetic expansion factor $\alpha=20$. Given the emitting area of the cathode, this resulted in an electron beam diameter of $12.5(7) \mathrm{mm}$ in the interaction section.

In all experiments, the cathode was illuminated using the 808-nm laser in transmission mode, so that electrons could thermalize with the GaAs lattice before extraction [27]. The temperature of the photocathode is not directly measured in operation. From off-line tests, it is known that the liquid nitrogen cooling system can reduce the GaAs temperature to $\lesssim 100 \mathrm{~K}$ when used at full cooling power. However, it has been observed that so low cathode temperatures lead to unstable emission currents-probably as cryo-adsorption then causes accelerated degradation of the $(\mathrm{Cs}, \mathrm{O})$ activation layer [21]. Normally, a reduced cold nitrogen flow was therefore used, for which, unfortunately, no temperature calibration exists, although it is clear that the cathodes must have been colder than room temperature. We assume that the cathode temperature was around $200 \mathrm{~K}$ in the experiments discussed here. Adopting the most conservative 
TABLE I. Operating parameters of the Electron Target, measured transverse cooling times $\tau_{\text {cool }}$, and normalized measured cooling rates $\lambda_{\exp }$ deduced from Eq. (19) for different molecular ions in the TSR. In all experiments, the photocathode operated at an estimated temperature of $\simeq 200 \mathrm{~K}$, and the beam was magnetically expanded by a factor $\alpha=20$ [Eq. (13)]. The column "betatron motion" indicates whether the horizontal $(x)$ or vertical $(y)$ betatron damping of the ion beam was evaluated. A label " $x+y$ " indicates that the cooling times for horizontal and vertical motions matched within their uncertainties, and that the given numbers are the average of both.

\begin{tabular}{lcccccccc}
\hline \hline Ion & $\begin{array}{c}\text { Ion } \\
\text { mass }(\mathrm{u})\end{array}$ & $\begin{array}{c}\text { Ion energy } \\
(\mathrm{MeV})\end{array}$ & $\begin{array}{c}\text { Electron } \\
\text { energy }(\mathrm{eV})\end{array}$ & $\begin{array}{c}\text { Electron } \\
\text { current }(\mu \mathrm{A})\end{array}$ & $\begin{array}{c}\text { Electron density } \\
\left(10^{6} \mathrm{~cm}^{-3}\right)\end{array}$ & $\begin{array}{c}\text { Exp. cooling } \\
\text { time } \tau_{\text {cool }}(\mathrm{s})\end{array}$ & $\begin{array}{c}\text { Exp. cooling } \\
\text { rate } \lambda_{\exp }\left(\mathrm{s}^{-1}\right)\end{array}$ & $\begin{array}{c}\text { Betatron } \\
\text { motion }(x / y)\end{array}$ \\
\hline $\mathrm{H}_{3}^{+}$ & 3 & 4.1 & 736 & 465 & $1.46(15)$ & $0.14(7)$ & $210(109)$ & $x+y$ \\
$\mathrm{HF}^{+}$ & 20 & 4.7 & 128 & 312 & $2.34(23)$ & $1.15(19)$ & $15.8(3.1)$ & $x$ \\
$\mathrm{DF}^{+}$ & 21 & 4.5 & 117 & 315 & $2.47(25)$ & $0.95(10)$ & $18.2(2.7)$ & $x$ \\
$\mathrm{~N}_{2} \mathrm{H}^{+}$ & 29 & 3.2 & 61 & 158 & $1.69(17)$ & $1.33(28)$ & $19.0(4.3)$ & $x+y$ \\
$\mathrm{DCO}^{+}$ & 30 & 3.1 & 51 & 273 & $3.19(32)$ & $1.21(45)$ & $13.2(3.2)$ & $x+y$ \\
$\mathrm{CF}^{+}$ & 31 & 2.6 & 44 & 235 & $2.95(30)$ & $1.38(14)$ & $10.6(1.2)$ & $x+y$ \\
$\mathrm{HS}^{+}$ & 33 & 2.7 & 44 & 219 & $2.78(28)$ & $1.12(21)$ & $13.8(2.8)$ & $x+y$ \\
${ }^{16} \mathrm{O}^{18} \mathrm{O}^{+}$ & 34 & 2.7 & 43 & 225 & $2.89(29)$ & $1.13(18)$ & $13.2(2.3)$ & $x+y$ \\
$\mathrm{H}^{35} \mathrm{Cl}^{+}$ & 36 & 2.4 & 37 & 219 & $3.00(30)$ & $1.93(58)$ & $7.4(2.1)$ & $x+y$ \\
$\mathrm{D}_{2}{ }^{37} \mathrm{Cl}^{+}$ & 41 & 2.3 & 31 & 169 & $2.49(25)$ & $3.3(2.3)$ & $5.2(3.7)$ \\
\hline \hline
\end{tabular}

uncertainty of $\pm 100 \mathrm{~K}$ for this estimate, and considering that relaxation processes can somewhat increase the electron temperature $T_{\text {cath }}$ upon emission to the vacuum [27], we expect an electron temperature $T_{e}$ after expansion [Eq. (13)] between $0.5 \mathrm{meV} / k_{\mathrm{B}}$ and $1.5 \mathrm{meV} / k_{\mathrm{B}}$, with a most likely value $T_{e} \simeq 1 \mathrm{meV} / k_{\mathrm{B}}$.

All ions under study had a (positive) charge $q=1$, while their masses ranged from $3 \mathrm{u}$ to $41 \mathrm{u}$. Operational parameters of TSR and Electron Target for the different experiments are given in columns 2-6 of Table I. For the heavier molecular ions $\left(m_{\mathrm{i}} \geq 30 \mathrm{u}\right)$, electron energies $\leq 51 \mathrm{eV}$ were required to match the low ion velocities. The storage ring always operated in coasting-beam mode (i.e., without rf-bunching), so that the ion velocity after cooling was defined by that of the electrons.

The gun acceleration voltages were greater than the given electron energies by approximately $10 \%$, accounting for the electron space charge potential and the difference in work functions between the stainless-steel beam pipe and the activated GaAs:(Cs,O) surface. Especially the latter effect is difficult to model. A calibration procedure that allows one to predict the true electron energy from the measured voltage and current of the electron gun in spite of this uncertainty has been developed as a preparation for CSR operation of the photocathode, where cooling times can be much longer [62]. In the TSR experiments, a coarse estimation of the space-charge and contact potential corrections was sufficient, followed by empirical finetuning of the acceleration voltage based on observation of the cooling process.

Typical emission currents from the photocathodes between 170 and $300 \mu \mathrm{A}$ were used for the heaviest ions, which is close to the Child-Langmuir limit of the electron gun for so low energies. With the magnetic expansion factor $\alpha=20$, this resulted in electron densities of the order of $10^{6} \mathrm{~cm}^{-3}$ as given in Table I.

\section{B. Data reduction}

For each experiment, we measured the transverse electron cooling times and the resulting final beam envelopes. The principle of the data analysis is illustrated in Figs. 3 and 4 on the examples of ${ }^{16} \mathrm{O}^{18} \mathrm{O}^{+}$and $\mathrm{HS}^{+}$. Both ion beams were produced from the Pelletron Van-de-Graaff accelerator at a kinetic energy of $2.7 \mathrm{MeV}$. No further (synchrotron) acceleration in the TSR was employed, so that electron cooling by the Target could start immediately after ion injection.

DR of the molecular ions led to neutral fragments that reached the imaging detector in the extraction beam line. For each recombination event, the c.m. of the fragments-and thus the hypothetical point of impact of the undissociated parent molecule - was reconstructed. Horizontal and vertical ion motions were analyzed separately. Binning of the singleparticle events according to detector hit coordinates and elapsed storage time yielded two-dimensional histograms as shown in Figs. 3 and 4 (upper left frames). Usually, the data of several equivalent ion injections was summed in order to improve counting statistics.

For each subdataset resulting from the chosen binning of the time axis, a Gaussian was fit to the distribution of c.m. coordinates to determine the standard deviation of the detector hits. These fits are shown for three particular storage times in the respective subframes (a), (b), and (c) of Figs. 3 and 4. One sees that the density of counts near the detector center increased as a function of storage time, although the integral ion current naturally decreased during the same time interval. As, in the regime of deep cooling, the recombination rates depend only on the dominating electron velocity spread [45], this effect must be explained by cooling of the remaining stored ions to higher transverse phase-space density.

Separately for the horizontal $(x)$ and vertical $(y)$ dimensions, the Gaussian fits yield the standard deviations $\hat{\sigma}_{x, y}(t)$ 

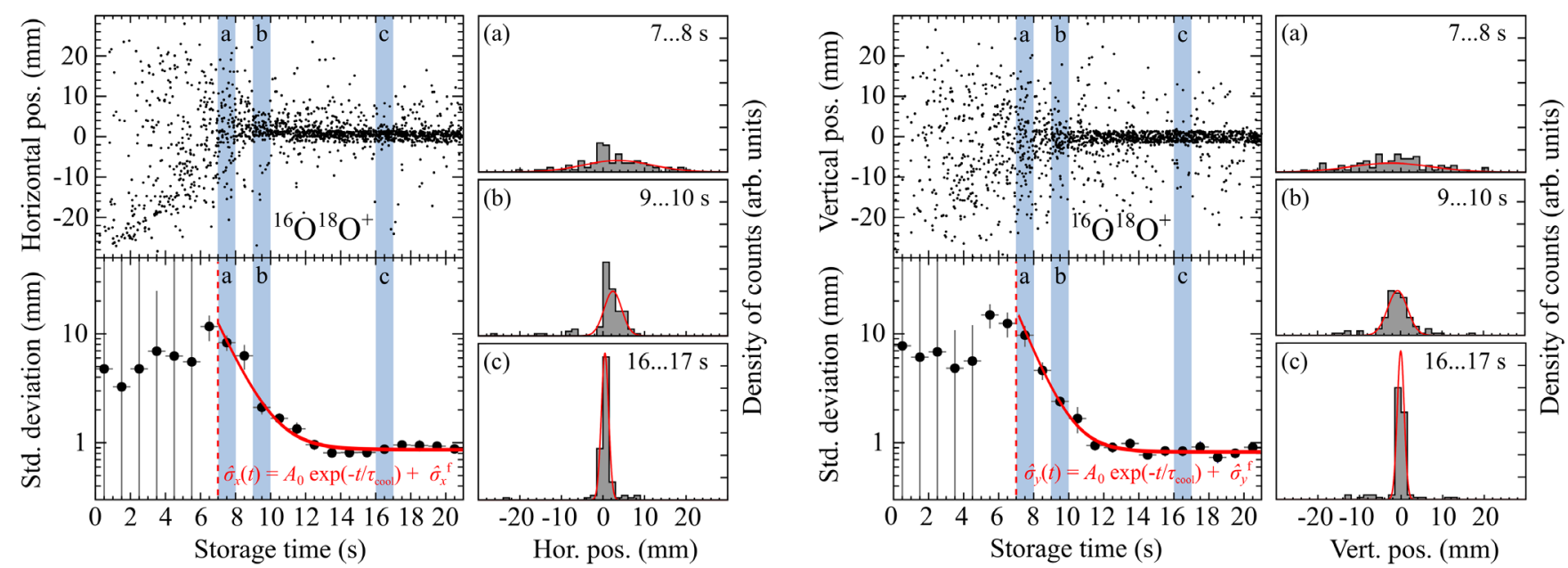

FIG. 3. Transverse electron cooling of ${ }^{16} \mathrm{O}^{18} \mathrm{O}^{+}$at $2.7 \mathrm{MeV}(79 \mathrm{keV} /$ nucleon). The left and right subfigures show the horizontal ( $x$ ) and vertical $(y)$ ion motions, respectively. Electron cooling started directly at ion injection ( $0 \mathrm{~s}$ storage time). Recombination of the molecular ion in the cooler led to neutral fragments ${ }^{16} \mathrm{O}$ and ${ }^{18} \mathrm{O}$ that were detected via neutral-fragment imaging. The top left frames show the distribution of the reconstructed centers-of-mass (c.m.) of these fragments as a function of storage time. The density of dots in the histogram is proportional to the density of hits on the detector. For 1-s time intervals, normal distributions were fit to the vertical and horizontal c.m. histograms, as exemplified in frames (a), (b), and (c). The obtained standard deviations are shown in the lower left frames. From $7 \mathrm{~s}$ of storage time, the evolution of the horizontal and vertical beam envelopes can be fit by an exponential decay (solid red curve), yielding the cooling times $\tau_{\text {cool }}$ and final envelope sizes $\hat{\sigma}^{\mathrm{f}}$ (see text).
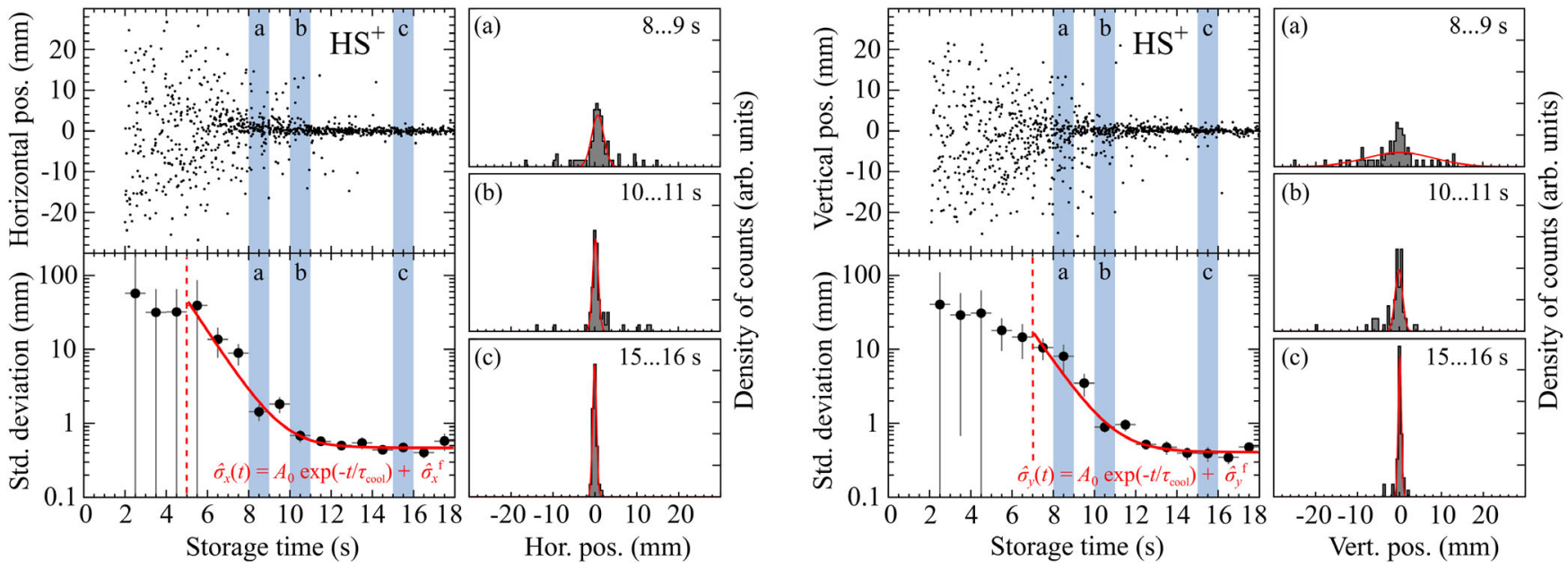

FIG. 4. Transverse electron cooling of $\mathrm{HS}^{+}$at $2.7 \mathrm{MeV}(81 \mathrm{keV} /$ nucleon $)$. Although electron cooling started directly at ion injection, data was recorded only for storage times $\geq 2$ s. The plots are analogous to the case of ${ }^{16} \mathrm{O}^{18} \mathrm{O}^{+}$from Fig. 3 .

of the detector hit coordinates as a function of storage time $t$. Via Eq. (3), $\hat{\sigma}_{x, y}$ is proportional to the ion beam size $\sigma_{x, y}$ in the Target.

As discussed in Sec. III, the transverse cooling process is divided into a slow and a fast phase, depending on the average ion velocities being large or small compared to the electron velocity spread. In our data, the slow phase of cooling is difficult to observe, as the initially large beam emittance led to scattering of the neutral products over practically the entire detector area, resulting in very large uncertainties on $\hat{\sigma}_{x, y}$. This can be seen in Figs. 3 and 4: in both measurements, the evolution of the beam envelopes cannot be followed clearly for the first $\sim 4 \ldots 5 \mathrm{~s}$ of storage time (note that, for $\mathrm{HS}^{+}$, data recording started only $2 \mathrm{~s}$ after injection, although electron cooling was enabled all the time). Once electron cooling has sufficiently decreased the emittance, the Gaussian fit provides a meaningful approximation of the product beam envelope, now much smaller than the detector aperture. From $\sim 6 \ldots 7 \mathrm{~s}$ onwards, exponential shrinking of $\hat{\sigma}_{x, y}$ to a final value below $1 \mathrm{~mm}$ is observed. We identify this with the phase of deep cooling. In this time window, the temporal evolution of the detectorhit standard deviation can be fit by an exponential decay $\hat{\sigma}(t)=A_{0} \exp \left(-t / \tau_{\text {cool }}\right)+\hat{\sigma}^{\text {f }}$ (once more, we omit 
the indices $x$ or $y$ for clarity). This yields, both, the experimental cooling time $\tau_{\text {cool }}$ as well as the final beam envelope at the detector $\hat{\sigma}^{\mathrm{f}}$ (cf. Figs. 3 and 4, lower left panes). In this, we assume that IBS heating $\left[\lambda_{\mathrm{IBS}}\right.$ in Eq. (14)] is negligible during the phase of exponential shrinking, and becomes relevant only just before the equilibrium beam size $\sigma^{\mathrm{f}}$ is reached. Because of the strong inverse dependence of IBS on the beam size $\left(\lambda_{\mathrm{IBS}} \sim \sigma^{-4.68}\right)$, this is a reasonable assumption.

For a typical dataset, the period of constant beam size is similarly long as the beam cooling phase, hence the fit results for $\tau_{\text {cool }}$ and $\hat{\sigma}^{\mathrm{f}}$ are practically independent of each other, and different temporal and spatial binning of the data can be used, depending on which fit parameter is of interest.

While $\hat{\sigma}^{\mathrm{f}}$ can be determined quite precisely, $\tau_{\text {cool }}$ is tied to a relatively large uncertainty for some of the experiments. This is because time-resolved analysis of the datasets was originally not foreseen. Resulting from short test measurements, performed in-between production runs, their intended purpose was merely to verify the good working condition of the electron cooler by observation of the final beam diameter, which is possible even at low numbers of counts. The resulting low statistics in the time-resolved analysis often makes it difficult to identify the onset of deep cooling. As a consequence, determination of the suitable region of interest for the exponential fit (indicated by vertical dashed red lines in Figs. 3 and 4) is linked to some uncertainty in itself that has been propagated to the results for $\tau_{\text {cool }}$.

\section{Cooling rates}

The so-measured cooling times $\tau_{\text {cool }}$ range from $\sim 0.1$ to $\sim 3 \mathrm{~s}$ for the different ions. For meaningful comparison of the experiments, we calculate normalized experimental cooling rates $\lambda_{\exp }$ as

$$
\lambda_{\exp }=\frac{1}{\tau_{\mathrm{cool}}} \frac{10^{6} \mathrm{~cm}^{-3}}{n_{e}} \frac{C}{l_{\mathrm{eff}}},
$$

where, following Eq. (12), we normalize to a typical electron density of $10^{6} \mathrm{~cm}^{-3}$ and cancel-out the scaling by the factor $l_{\text {eff }} / C$ in order to obtain results that are independent on the geometry of our particular setup.

The results from all experiments are summarized in the last three columns of Table I. For most ion beams, very similar cooling times were found for the horizontal and vertical betatron motions. In these cases, indicated by labels " $x+y$ " in Table I, the given values of $\tau_{\text {cool }}$ and $\lambda_{\exp }$ are averaged over the two degrees of freedom. In three experiments $\left(\mathrm{HF}^{+}, \mathrm{DF}^{+}\right.$and $\left.\mathrm{D}_{2}{ }^{37} \mathrm{Cl}^{+}\right)$, cooling of the vertical betatron motion could not be measured, as data acquisition of the fragment imaging detectors started only after the vertical cooling process was practically complete. In the horizontal dimension, however, the onset of deep cooling was delayed-probably because of the larger initial horizontal emittance after multiturn injection-so that it was still observable in the data. For these experiments, only the horizontal cooling rate was evaluated, as indicated by the labels " $x$ " in Table I.

By the normalization of Eq. (19) we remove the straightforward linear dependence of the cooling rate on the electron density. Such a pure proportionality would be expected if both, the transverse electron temperature $T_{e}$ and the Coulomb logarithm $L_{C}$ were completely independent of $n_{e}$. While no dedicated experiments were carried out to probe this independence, it is supported to hold in good approximation by the analysis of the available data.

The effective $n_{e}^{-1}$-dependence of the cooling time $\tau_{\text {cool }}$ could be verified via three experiments on $\mathrm{CF}^{+}$. Except for small differences in ion energy ( $2.6 \mathrm{MeV}$ vs $2.8 \mathrm{MeV}$ ), the same ion beam was electron cooled using three different emission currents from the photocathode, at otherwise identical operating parameters of the electron target. Figure 5 shows the measured cooling times $\tau_{\text {cool }}$ as a function the corresponding electron densities $n_{e}$. The dotted black curve is a fit of a simplified $\tau_{\text {cool }} \sim n_{e}^{-1}$ law as in Eq. (12). The other curves show fits of Eq. (11) with $\lambda$ taken from the full binary collision models for flattened [dashed-blue curve, Eq. (9)] and isotropic [solid red, Eq. (8)] velocity distributions. The latter two models thus take the density dependence of $L_{C}$ from Eq. (10) into account. The transverse electron temperature $T_{e}$ is the only free parameter and fits at $T_{e}=0.86(4) \mathrm{meV} / k_{\mathrm{B}}$

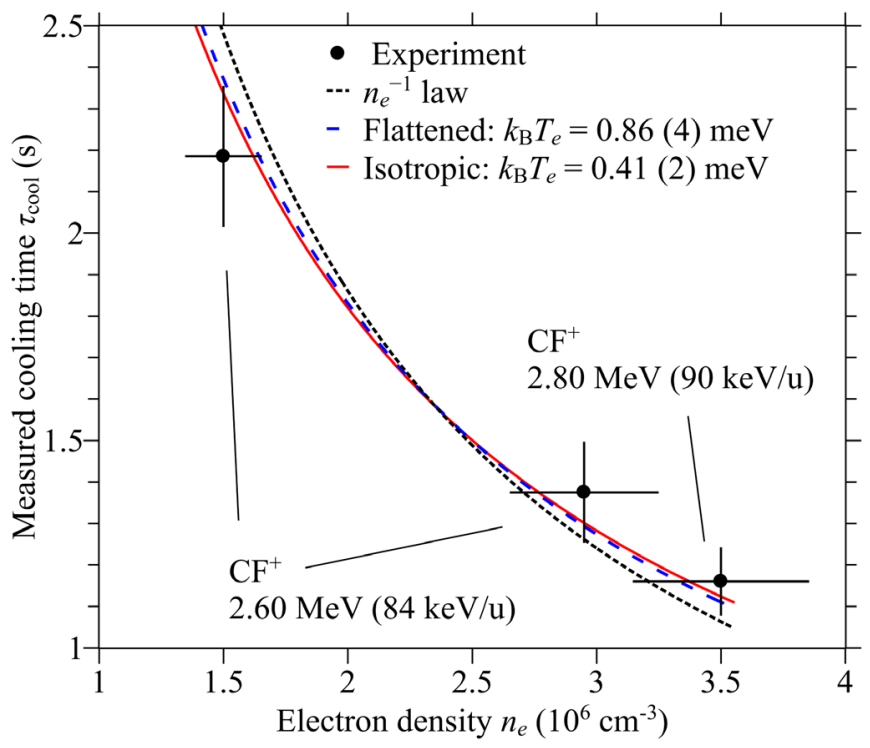

FIG. 5. Measured transverse cooling times $\tau_{\text {cool }}$ for beams of $\mathrm{CF}^{+}\left(m_{\mathrm{i}}=31 \mathrm{u}\right)$ as a function of the electron density $n_{e}$. The short-dashed black curve is a simplified $1 / n_{e}$-dependence as in Eq. (12). The other curves are fits of Eq. (11) using the cooling rates $\lambda$ from Eqs. (9) (long-dashed blue) and (8) (solid red), respectively, thus taking the density- and temperature-dependence of $L_{C}$ into account. 
$(10.0 \pm 0.4 \mathrm{~K})$ and $T_{e}=0.41(2) \mathrm{meV} / k_{\mathrm{B}}(4.7 \pm 0.2 \mathrm{~K})$ for the flattened and isotropic models, respectively.

As visible in Fig. 5, differences between the three models are hardly relevant, considering the statistical uncertainty of the data. By extrapolation, we estimate that the systematic error introduced in our $\lambda_{\exp }$ by assumption of a constant value of $L_{C}$ is no larger than $10 \%$.

The assumption of a constant electron temperature $T_{e}$ in all experiments from Table I is justified if Eq. (13) holds, i.e., if $T_{e}$ is defined by the cathode temperature $T_{\text {cath }}$ and the magnetic expansion factor $\alpha$ only. If so, an increase of the expansion factor has two counteracting effects on the transverse cooling time [cf. Eq. (12)]: At fixed total electron current, the electron density $n_{e}$ decreases $\sim \alpha^{-1}$ due to the rising cross section area of the beam, which, in itself, would lead to an increase of the cooling time. However, also $T_{e}$ decreases $\sim \alpha^{-1}$ and outweighs the effect of reduced density in Eq. (12) so that, as a net effect, $\tau_{\text {cool }}$ decreases $\sim \alpha^{-1 / 2}$.

Figure 6 shows the measured cooling time for a beam of $\mathrm{HF}^{+}$, stored at $4.7 \mathrm{MeV}(233 \mathrm{keV} /$ nucleon $)$, as a function of the magnetic expansion factor $\alpha$. The magnetic field at the electron gun was increased from the nominal value of $0.8 \mathrm{~T}$ to $1.2 \mathrm{~T}$ and $1.6 \mathrm{~T}$. The guiding field in the interaction section of the target was kept fixed at the usual value of $0.04 \mathrm{~T}$, resulting in values of $\alpha=20,30$ and 40. The electron kinetic energy and the emission current of the electron gun were held at the values given in Table I.

Again, the dotted black curve is a fit of the simplified $\alpha^{-1 / 2}$ law motivated above, while the dashed-blue and solid red curves are fits of the full binary collision models defined by Eq. (11) in combination with the "flattened" and "isotropic" cooling rates from Eqs. (9) and (8), respectively. $T_{e}$ has been substituted using Eq. (13), so that, this time, $T_{\text {cath }}$ was the only free parameter in the latter two fits.

For the isotropic model, we find $T_{\text {cath }}=88 \pm 10 \mathrm{~K}$. As noted in Sec. IVA, this result is somewhat unrealistic, as cathode temperatures close to the boiling point of nitrogen are known to cause unstable emission currents [21]. Using the cooling rate for a flattened velocity distribution yields $T_{\text {cath }}=193 \pm 17 \mathrm{~K}$ which agrees better with the previous estimates.

Overall, the measurement agrees quite well with the models. The fact that no rise in the cooling time is observed with increasing $\alpha$ supports the interpretation that the density-related weakening of the cooling force was indeed over-compensated by the lower transverse temperature $T_{e}$ after stronger expansion. We note that, in the given lowvoltage operation of the Electron Target, expansion took place only after acceleration of the electrons to their final energy, hence the longitudinal electron temperaturedefined by (density-dependent) plasma relaxation effects $[3,56]$ - is expected to have been constant in all three cases.

For the normally-used electron beam expansion $\alpha=20$, Fig. 7 shows the measured normalized cooling rates $\lambda_{\exp }$ from all experiments of Table I as a function of ion mass $m_{\mathrm{i}}$.

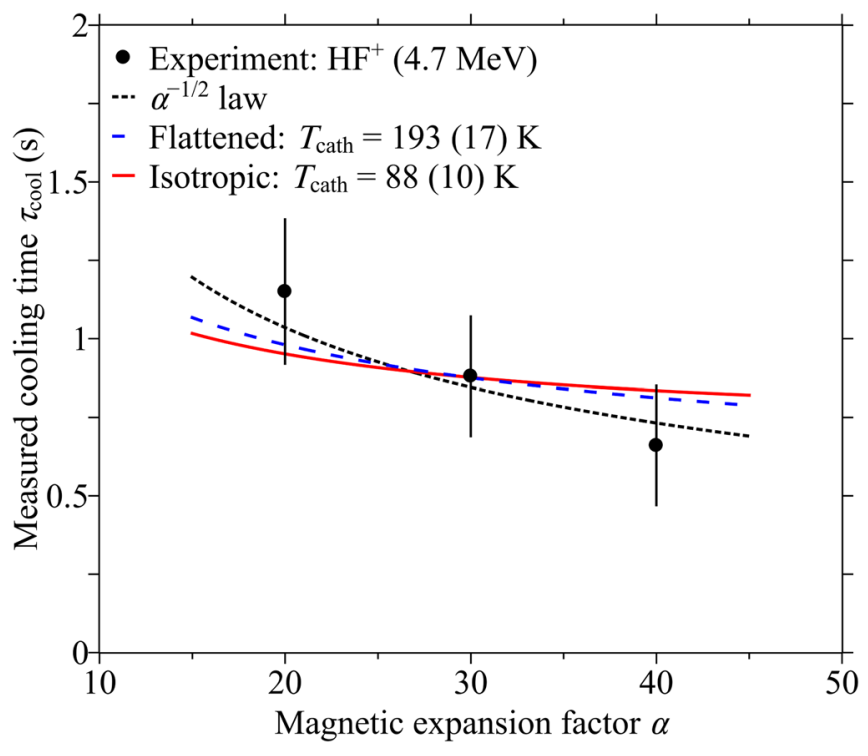

FIG. 6. Cooling time $\tau_{\text {cool }}$ for $\mathrm{HF}^{+}$at $4.7-\mathrm{MeV}(233 \mathrm{keV} /$ nucleon) as a function on the expansion factor $\alpha$ of the electron beam, with other Target parameters held fixed. Only the horizontal component of the betatron motion has been analyzed. The data is compared to a simplified $\alpha^{-1 / 2}$-law (short-dashed black curve) and to the models from Eqs. (9) (long-dashed blue) and (8) (solid red) as described in the text.

The shaded areas indicate the expected cooling rates for effective electron temperatures $T_{e}$ between $0.5 \mathrm{meV} / k_{\mathrm{B}}$ (upper bound) and $1.5 \mathrm{meV} / k_{\mathrm{B}}$ (lower bound), calculated for flattened [Eq. (9), dotted blue] and isotropic [Eq. (8), dashed red] electron velocity distributions, respectively.

The dotted black curve is a fit of the experimental data to a $1 / m_{\mathrm{i}}$-law as expected from Eq. (12). The single fit coefficient corresponds to an average $T_{e}$ of $0.92(5) \mathrm{meV} / \mathrm{k}_{\mathrm{B}}$ $(10.7 \pm 0.6 \mathrm{~K})$ or $0.45(3) \mathrm{meV} / k_{\mathrm{B}}(5.2 \pm 0.3 \mathrm{~K})$ for the "flattened" and "isotropic" cases, respectively. The good agreement with the expected $1 / m_{\mathrm{i}}$-dependence suggests that electron cooling could be used efficiently over the entire range of ion masses from $3 \mathrm{u}$ to $41 \mathrm{u}$. Again, the "flattened" model provides a more useful prediction of the measured cooling rates based on our above estimate of $\simeq 1 \mathrm{meV} / k_{\mathrm{B}}$ for the transverse electron temperature.

We note that, in resonant recombination of $\mathrm{Sc}^{18+}$, Lestinsky et al. observed a transverse electron temperature of $1.1(1) \mathrm{meV} / k_{\mathrm{B}}$, the lowest value reported for the Electron Target in that kind of experiment [35]. In an effort to achieve highest energy resolution, they cooled the photocathode to a lower temperature $(\simeq 110 \mathrm{~K})$ and used somewhat stronger magnetic expansion $(\alpha=28)$. However, because of the much higher electron beam energy, their experiment is more sensitive to virtual increase of $T_{e}$ by field imperfections [59], which may explain why their effective value of $T_{e}$ is so similar to ours.

The fact that our effective transverse temperature deduced from the observed cooling rates agrees relatively 


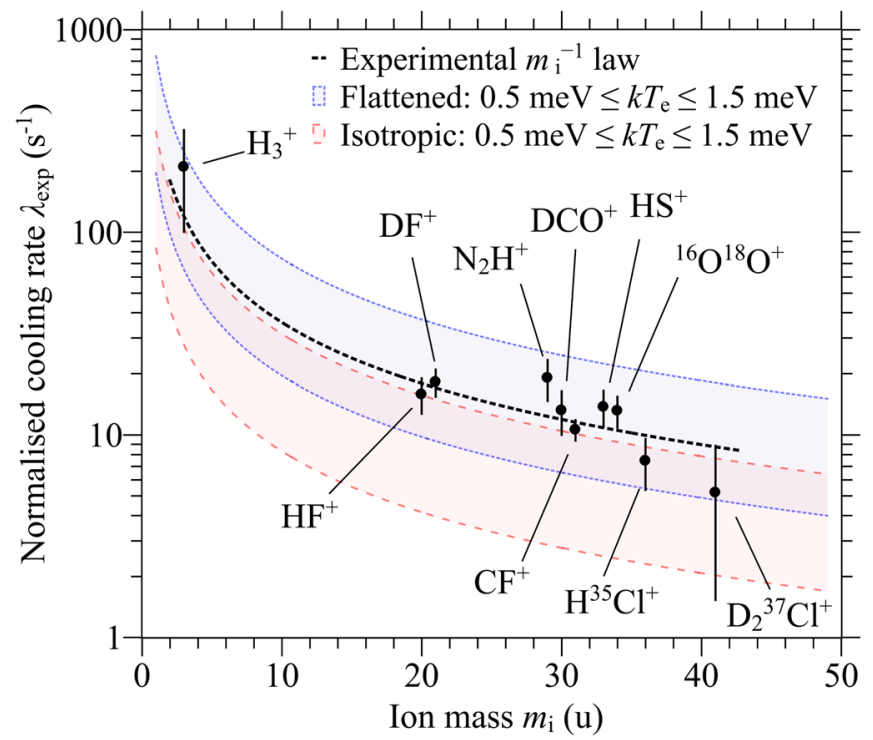

FIG. 7. Overview of the measured normalized cooling rates for magnetic expansion $\alpha=20$. The dots represent the data from Table I. The dotted-black curve is a fit of the measurement to a $1 / m_{\mathrm{i}}$-model as expected from Eq. (12). The shaded areas delimit the expected rates in the electron temperature interval $0.5 \mathrm{meV} / k_{\mathrm{B}} \leq T_{e} \leq 1.5 \mathrm{meV} / k_{\mathrm{B}}$ for a flattened and isotropic electron velocity distribution, as indicated.

well with the value obtained from recombination measurements may come as a surprise, as one could have expected that the magnetic field-neglected in our simple cooling model-would increase the damping rates at least somewhat, while not affecting the resolution of recombination experiments [48]. Based on our data, we are unable to explain that divide between our experiments and more recent magnetized cooling models. However, we note that the latter have mostly been probed against direct measurements of the longitudinal cooling force as a function of the discrete relative ion-electron velocity $u[6,50,52]$. For the transverse cooling process - that is most often observed only as a statistical evolution of the incoherent ion ensemble-fewer and arguably less precise measurements have been published. While some experiments did observe a dependence of the transverse cooling rate on the magnetic field [63], absolute cooling times have often been reported to agree quite well with a simple nonmagnetic model, as in our case $[64,65]$. In fact, early experimental studies of magnetized cooling noted that the overall rate enhancement by the magnetic field may be less obvious for the transverse directions because of higher-order effects in the electronion interaction [49].

\section{Final beam quality}

Via the methods laid out in Secs. II and III, the steadystate spreads $\hat{\sigma}^{\mathrm{f}}$ of the neutral c.m. distribution give information about the achieved ion beam quality. Using Eq. (3), the ion beam sizes $\sigma_{x, y}$ in the Electron Target can be computed which, in-turn, are connected to the transverse emittances $\varepsilon_{x, y}^{95 \%}$ and velocity spreads $\sigma_{x, y}^{v}$ via Eqs. (4) and (17). The so-derived steady-state beam properties are listed in Table II.

In most cases, $68 \%$ beam diameters $2 \sigma \lesssim 1 \mathrm{~mm}$ are achieved for both planes. The horizontal beam envelope is always wider than the vertical one, as expected from the different values of the beta functions at the Electron Target (cf. Fig. 1). Assuming equal horizontal and vertical cooling forces, the equilibrium ion beam divergences $\sigma_{x}^{\prime}$ and $\sigma_{y}^{\prime}$ should be equal as well. From Eqs. (4) we thus expect

$$
\sigma_{y}=\frac{\beta_{y}}{\beta_{x}} \sigma_{x} .
$$

As shown in Fig. 8, the measured aspect ratios of the beam cross sections agree very well with this simple reasoning. Although the Electron Target's nonzero dispersion $\left(D_{x}=2.0 \mathrm{~m}\right)$ opens up the possibility of dispersive cooling, which can enhance the horizontal cooling rate compared to the vertical one [66], such asymmetry is

TABLE II. Ion beam properties after electron cooling. Equilibrium beam sizes, velocity spreads and final emittances are derived from the measured steady-state envelope sizes $\hat{\sigma}^{\mathrm{f}}$ of the neutral-product beams at the particle detectors using Eqs. (3), (17) and (4).

\begin{tabular}{|c|c|c|c|c|c|c|c|c|c|c|}
\hline \multirow[b]{2}{*}{ Ion } & \multirow{2}{*}{$\begin{array}{c}\text { Mass } \\
m_{\mathrm{i}}(\mathrm{u})\end{array}$} & \multirow{2}{*}{$\begin{array}{l}\text { Energy } \\
(\mathrm{MeV})\end{array}$} & \multirow{2}{*}{$\begin{array}{l}\text { Velocity } \\
(c)\end{array}$} & \multirow{2}{*}{$\begin{array}{c}\text { Revolution } \\
\text { frequency } \\
f_{0}\left(10^{3} \mathrm{~s}^{-1}\right)\end{array}$} & \multicolumn{2}{|c|}{$68 \%$ diameter $2 \sigma(\mathrm{mm})$} & \multicolumn{2}{|c|}{ Velocity spread $\sigma^{v}(\mathrm{~m} / \mathrm{s})$} & \multicolumn{2}{|c|}{$95 \%$ emittance $\varepsilon^{95 \%}(\mathrm{~nm})$} \\
\hline & & & & & $x$ & $y$ & $x$ & $y$ & $x$ & $y$ \\
\hline $\mathrm{H}_{3}^{+}$ & 3 & 4.1 & 0.054 & 290 & $0.16(2)$ & $0.08(1)$ & $212(24)$ & $284(26)$ & $4.2(7)$ & $2.9(4)$ \\
\hline $\mathrm{HF}^{+}$ & 20 & 4.7 & 0.022 & 121 & $0.21(3)$ & $0.07(1)$ & $118(15)$ & $108(16)$ & $7.4(1.3)$ & $2.4(5)$ \\
\hline $\mathrm{DF}^{+}$ & 21 & 4.5 & 0.021 & 116 & $0.26(2)$ & $0.08(1)$ & $141(9)$ & $113(15)$ & $12(1)$ & $2.9(6)$ \\
\hline $\mathrm{N}_{2} \mathrm{H}^{+}$ & 29 & 3.2 & 0.015 & 84 & $0.53(14)$ & $0.23(9)$ & $205(53)$ & $235(89)$ & $47(17)$ & $24(13)$ \\
\hline $\mathrm{DCO}^{+}$ & 30 & 3.1 & 0.014 & 76 & $0.55(8)$ & $0.25(2)$ & $195(28)$ & $227(22)$ & $51(11)$ & $27(4)$ \\
\hline $\mathrm{CF}^{+}$ & 31 & 2.6 & 0.013 & 71 & $0.37(2)$ & 0.19 (1) & $121(6)$ & $164(10)$ & $23(2)$ & $16(2)$ \\
\hline $\mathrm{HS}^{+}$ & 33 & 2.7 & 0.013 & 71 & $0.40(4)$ & $0.15(2)$ & 133 (12) & $126(13)$ & 27 (3) & $9.5(1.3)$ \\
\hline${ }^{16} \mathrm{O}^{18} \mathrm{O}^{+}$ & 34 & 2.7 & 0.013 & 70 & $0.62(9)$ & $0.22(3)$ & 200 (29) & $187(25)$ & $63(13)$ & $21(4)$ \\
\hline $\mathrm{H}^{35} \mathrm{Cl}^{+}$ & 36 & 2.4 & 0.012 & 65 & $0.9(3)$ & $0.4(2)$ & 265 (79) & $290(116)$ & $129(55)$ & $59(34)$ \\
\hline $\mathrm{D}_{2}{ }^{37} \mathrm{Cl}^{+}$ & 41 & 2.3 & 0.011 & 60 & $1.7(3)$ & $0.6(1)$ & 467 (88) & $435(67)$ & 479 (127) & $160(35)$ \\
\hline
\end{tabular}




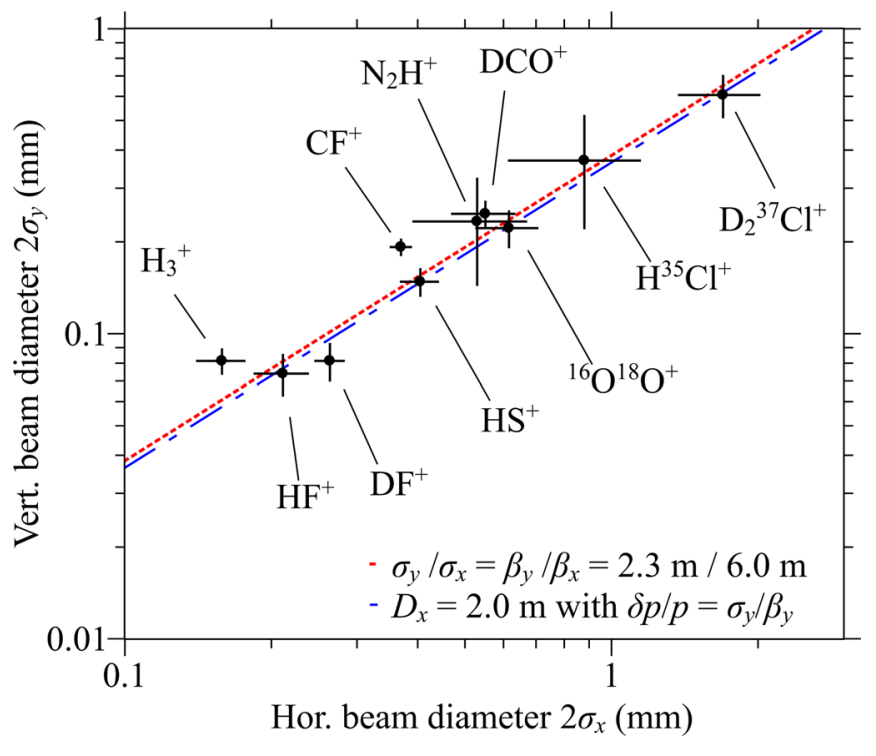

FIG. 8. Steady-state beam diameters (68\% envelopes) after electron cooling. The black dots represent the data from Table II. The dashed red line indicates the expected ratio of diameters at equal horizontal and vertical divergence, given the amplitudes of the beta functions of the TSR at the Electron Target (see also Fig. 1). The blue dashed curve also accounts for the dispersion $\left(D_{x}=2.0 \mathrm{~m}\right)$ in the Target, assuming a momentum spread $\delta p / p=\sigma_{y} / \beta_{y}$ (see text).

visible—if at all—only for two experiments: $\mathrm{H}_{3}^{+}$and $\mathrm{CF}^{+}$. All other measurements align perfectly with Eq. (20). A possible explanation lies in the fact that the standard working point of TSR is very close to the first-order coupling resonance [30], so that horizontal and vertical betatron motions are entangled, and heating and cooling effects averaged between the two degrees of freedom.

As discussed in Sec. II C, the nonzero dispersion in the Target could additionally contribute to the total horizontal beam width $\sigma_{x}^{\text {tot }}$ as given by Eq. (5). Using the transverse divergence as a conservative estimate for the momentum spread $\delta p / p$, Eq. (20) changes into $\sigma_{y}=\left(\beta_{y} / \beta_{x}\right) \sigma_{x}^{\text {tot }} / \sqrt{1+D_{x}^{2} / \beta_{x}^{2}}$. This is visualized as the blue dash-dotted-curve in Fig. 8. As noted before, the effect of dispersion on the horizontal diameter of the electroncooled beam is insignificant.

Already from Fig. 8 it appears that heavier ions have larger final beam diameters. This may surprise at first, as the IBS heating rate $\left(\sim m_{\mathrm{i}}^{-2}\right)$ decreases faster with mass than the cooling rate $\left(\sim m_{\mathrm{i}}^{-1}\right)$. Unfortunately, the heavier ions also have lower revolution frequencies in the TSR, so that the velocity-dependence $\sim\left(f_{0} C\right)^{-3 / \gamma}$ in Eq. (16) outweighs the favorable effect of mass. Figure 9 shows the measured final beam diameters as compared to an estimate for the IBS-limited horizontal beam size according to Eq. (16) (green-shaded area). The latter was calculated using the measured cooling times $\tau_{\text {cool }}$ and revolution frequencies $f_{0}$ for each ion species. The number of stored ions $N_{\mathrm{i}}$ certainly

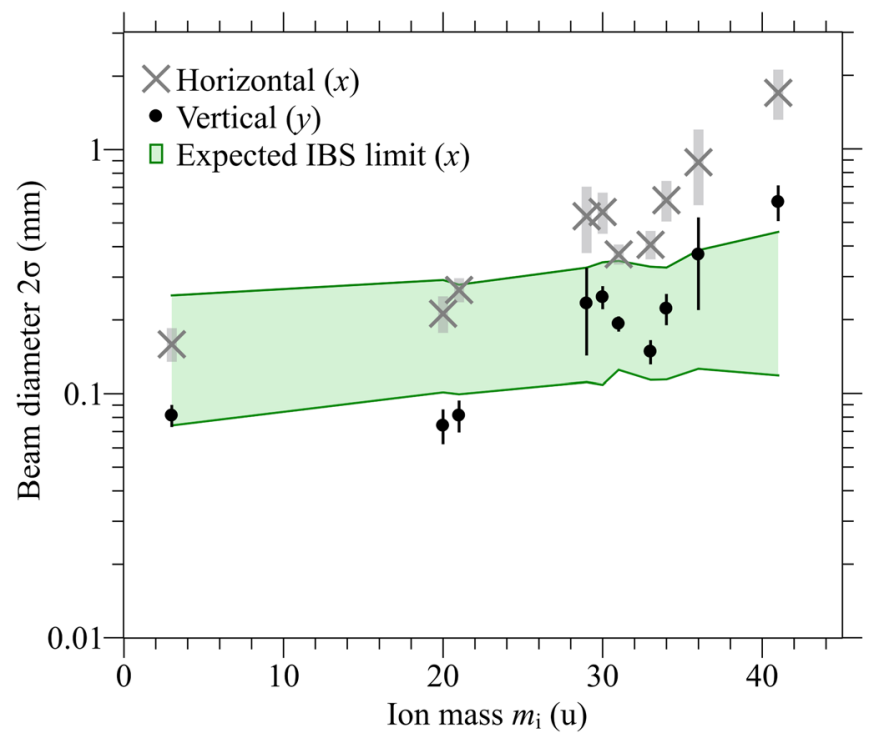

FIG. 9. Steady-state horizontal $(x)$ and vertical (y) beam diameters $2 \sigma$ as a function of ion mass. The shaded green area shows the span of expected horizontal beam diameters in equilibrium between electron cooling and IBS heating [Eq. (16)], given the observed $\tau_{\text {cool }}$. The upper and lower limits correspond to $10^{6}$ and $10^{4}$ stored ions, respectively.

varied strongly among the experiments (and as a function of storage time), hence it is difficult to estimate precisely. From the known acceptance limit of the detector systems and the usual electron recombination rate coefficients, we estimate a typical ion number $10^{4} \leq N_{\mathrm{i}} \leq 10^{6}$. The lower and upper bounds of the shaded area correspond to these limits, combined with the experimental uncertainty of $\tau_{\text {cool }}$. The coefficient $a_{x} \simeq 4 \times 10^{-13} \mathrm{~mm}^{\gamma} / \mathrm{s}$ in Eq. (15) is obtained from measurements of the horizontal IBS process (Fig. 3 of Artikova et al. [61]), hence also Eq. (16) is strictly valid only for the horizontal dimension.

For the lighter ions, the directly measured beam envelopes agree with the predicted IBS limit. While the latter does in fact increase smoothly with rising ion mass, the model cannot explain the significantly larger beam diameters obtained for some of the heavier ions $\left(m_{\mathrm{i}} \gtrsim 30 \mathrm{u}\right)$.

The measured ion velocity spreads (cf. Table II) are indeed much smaller than the transverse electron velocities that should have been in the order of $10^{4} \mathrm{~m} / \mathrm{s}$ for $T_{e} \simeq 1 \mathrm{meV} / k_{\mathrm{B}}$. Assuming that they stem from thermal population of the betatron oscillations, transverse ion beam temperatures in the Target can be computed according to Eq. (18). These are plotted in Fig. 10 together with the average electron temperatures obtained from the cooling rates (Fig. 7) under assumption of the "flattened" (blue) and "isotropic" (red) binary collision model, respectively. Again, the green shaded area shows the expectation for equilibrium between electron cooling and IBS heating. The IBS-limited beam size was calculated from Eq. (16) analogously to Fig. 9 and converted into an ion temperature via 


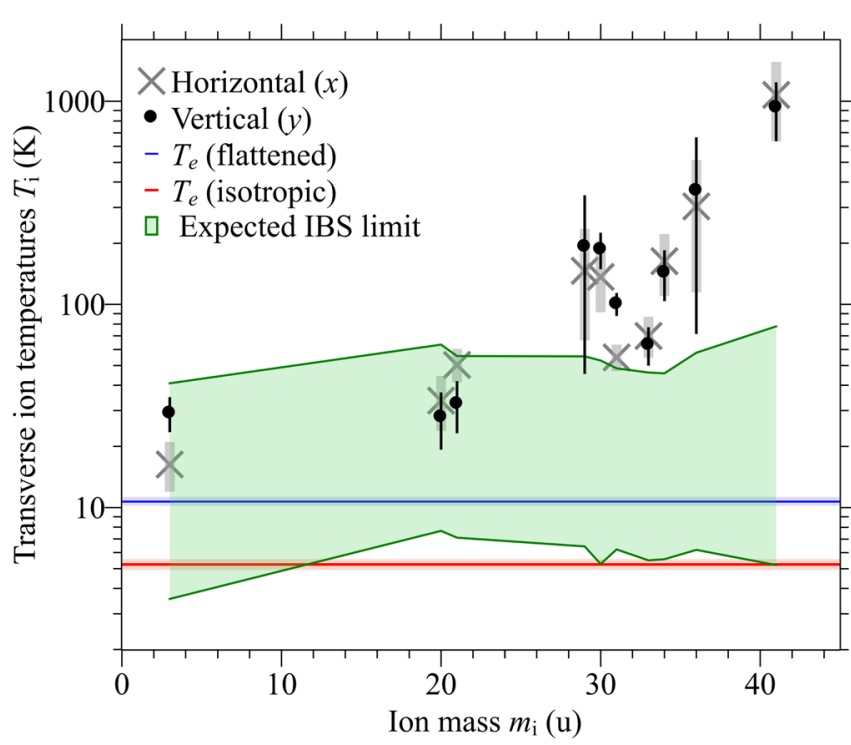

FIG. 10. Horizontal and vertical transverse ion beam temperatures after electron cooling. The colored horizontal bars indicate the best-fit electron temperatures $T_{e}$ corresponding to the observed cooling rates, for flat and isotropic velocity distributions. The shaded green area is the expected ion temperature corresponding to the IBS limit (see text).

Eq. (18). Also this plot shows that the IBS model provides a reasonable prediction for the lightest ions, while the heavier ones have significantly higher temperatures.

The beam quality thus seems close to optimal for the lower half of the ion mass range, but worsens more strongly than expected for $m_{\mathrm{i}} \gtrsim 30$. The reason for this is difficult to identify. An explanation may lie in the fact that, with longer cooling times, adjustment of the electron cooler becomes more difficult and time-consuming for the operator, so that cooler and ion beams are less likely to have been optimally aligned. This may have resulted in small angles $\sim 1 \mathrm{mrad}$ between the electron and ion beams. At the low beam velocities, the transverse collision energy related to such a misalignment is much smaller than $T_{e}$, hence cooling is still expected to work overall in that situation. However, recent numerical simulations of electron cooling at ELENA suggest that even angular misalignment smaller than the thermal divergence of the electron beam can lead to a notable increase of the final ion emittance [67].

In spite of these beam envelopes slightly larger than expected, Table II shows that $95 \%$ transverse beam emittances $\varepsilon^{95 \%}$ much better than $1 \mu \mathrm{m}$ were obtained in all experiments. In fact, values of $\varepsilon^{95 \%}$ in the order of $10 \mathrm{~nm}$ have been measured even for some of the heavier ions.

\section{SUMMARY AND OUTLOOK}

By analysis of a series of past TSR experiments, we have shown that electron cooling has been applied successfully to singly-charged ions of masses up to $41 \mathrm{u}$. Limited by the magnetic rigidity of the TSR, the velocities of the stored ions were very low, and the corresponding electron energies reached down to $31 \mathrm{eV}$. While the low electron energy does not cause problems by itself, the low extraction voltage limits the emission current of the electron gun. Under those circumstances, a cold GaAs:(Cs,O) photocathode electron source could be employed without further limiting the electron density in the cooler, but with the advantage of much lower transverse electron beam temperature.

After several seconds of cooling time, high-quality beams of $95 \%$ transverse emittance $\ll 1 \mu \mathrm{m}$ could be delivered to experiments. The observed transverse cooling rates can be modeled quite well using a simple nonmagnetic binary collision model assuming a flattened electron velocity distribution and an effective transverse electron temperature $T_{e} \simeq 1 \mathrm{meV} / k_{\mathrm{B}}$. The latter agrees well with the value expected from the usual operating parameters of the electron gun.

With the focus of research at MPIK shifting toward everheavier molecular ions, both the TSR and its injection accelerators were decommissioned end of 2012. However, the cold electron gun and its related facilities for handling of the GaAs photocathodes continue to operate as a part of the newer CSR facility [25]. In fact, experience gathered in operation of the TSR Electron Target had a significant impact on the design of the CSR electron cooler. Aiming at even heavier singly-charged molecular ions and velocitylimited by the maximum rigidity of the purely electrostatic ring, it has to operate at electron energies and densities still considerably lower than those reported here [26]. The present analysis will therefore serve as a valuable benchmark when exploring the possibilities of electron cooling at the CSR.

\section{ACKNOWLEDGMENTS}

This work received financial support by the Max Planck Society for the Advancement of Science (MPG), as well as invaluable technical support from all members of the MPIK accelerator staff, which we hereby gratefully acknowledge. We gratefully recognize the invaluable steady support of photocathode electron cooling development at MPIK by the late Dirk Schwalm.

[1] G. I. Budker, N. S. Dikansky, V. I. Kudelainen, I. N. Meshkov, V. V. Parchomchuk, D. V. Pestrikov, A. N. Skrinsky, and B. N. Sukhina, Part. Accel. 7, 197 (1976).

[2] A. Wolf, G. Gwinner, J. Linkemann, A. A. Saghiri, M. Schmitt, D. Schwalm, M. Grieser, M. Beutelspacher, T. Bartsch, C. Brandau, A. Hoffknecht, A. Müller, S. Schippers, O. Uwira, and D. W. Savin, Nucl. Instrum. Methods Phys. Res., Sect. A 441, 183 (2000).

[3] H. Danared, A. Källberg, G. Andler, L. Bagge, F. Österdahl, A. Paál, K.-G. Rensfelt, A. Simonsson, Ö. Skeppstedt, and M. af Ugglas, Nucl. Instrum. Methods Phys. Res., Sect. A 441, 123 (2000). 
[4] H. Poth, Nature (London) 345, 399 (1990).

[5] A. Wolf, C. Ellert, M. Grieser, D. Habs, B. Hochadel, R. Repnow, and D. Schwalm, Beam cooling and related topics, CERN Report No. 94-03, 1994, pp. 416-421, https://cds.cern.ch/record/254427/files/CERN-94-03.pdf.

[6] T. Winkler, K. Beckert, F. Bosch, H. Eickhoff, B. Franzke, O. Klepper, F. Nolden, H. Reich, B. Schlitt, P. Spädtke, and M. Steck, Hyperfine Interact. 99, 277 (1996).

[7] O. Novotný, O. Motapon, M. H. Berg, D. Bing, H. Buhr, H. Fadil, M. Grieser, J. Hoffmann, A. S. Jaroshevich, B. Jordon-Thaden, C. Krantz, M. Lange, M. Lestinsky, M. Mendes, S. Novotny, D. A. Orlov, A. Petrignani, I. F. Schneider, A. E. Orel, and A. Wolf, J. Phys. Conf. Ser. 192, 012021 (2009).

[8] J. Stützel, O. Novotný, S. Novotny, D. A. Orlov, H. Buhr, M. B. Mendes, C. Greene, J. Hoffmann, C. Krantz, S. Altevogt, M. H. Berg, D. Bing, M. Froese, M. Grieser, B. Jordon-Thaden, M. Lange, M. Lestinsky, A. Petrignani, A. Shornikov, and A. Wolf, J. Phys. Conf. Ser. 194, 062028 (2009).

[9] H. Kreckel, O. Novotný, K. N. Crabtree, H. Buhr, A. Petrignani, B. A. Tom, R. D. Thomas, M. H. Berg, D. Bing, M. Grieser, C. Krantz, M. Lestinsky, M. B. Mendes, C. Nordhorn, R. Repnow, J. Stützel, A. Wolf, and B. J. McCall, Phys. Rev. A 82, 042715 (2010).

[10] J. Stützel, Fragmentation of small multi-electron molecular ions in cold electron collisions, Dissertation, Heidelberg, 2010, https://doi.org/10.11588/heidok.00011597.

[11] A. Petrignani, S. Altevogt, M. H. Berg, D. Bing, M. Grieser, J. Hoffmann, B. Jordon-Thaden, C. Krantz, M. B. Mendes, O. Novotný, S. Novotny, D. A. Orlov, R. Repnow, T. Sorg, J. Stützel, A. Wolf, H. Buhr, H. Kreckel, V. Kokoouline, and C. H. Greene, Phys. Rev. A 83, 032711 (2011).

[12] C. Nordhorn, D. Bing, H. Buhr, M. Grieser, O. Heber, C. Krantz, M. B. Mendes, R. Repnow, D. Schwalm, A. Shornikov, J. Stützel, and A. Wolf, J. Phys. Conf. Ser. 300, 012004 (2011).

[13] O. Novotný, A. Becker, H. Buhr, C. Domesle, W. Geppert, M. Grieser, C. Krantz, H. Kreckel, R. Repnow, D. Schwalm, K. Spruck, J. Stützel, B. Yang, A. Wolf, and D. W. Savin, Astrophys. J. 777, 54 (2013).

[14] A. Becker, C. Domesle, W. D. Geppert, M. Grieser, C. Krantz, R. Repnow, D. W. Savin, D. Schwalm, A. Wolf, B. Yang, and O. Novotný, J. Phys. Conf. Ser. 635, 072067 (2015).

[15] D. O. Kashinsky, D. Talbi, A. P. Hickman, O. E. Di Nallo, F. Colboc, K. Chakrabarti, I. F. Schneider, and J. Z. Mezei, J. Chem. Phys. 146, 204109 (2017).

[16] O. Novotný, H. Buhr, W. Geppert, M. Grieser, M. Hamberg, C. Krantz, M. B. Mendes, A. Petrignani, R. Repnow, D. W. Savin, D. Schwalm, J. Stützel, and A. Wolf, Astrophys. J. 862, 166 (2018).

[17] D. Habs et al., Nucl. Instrum. Methods Phys. Res., Sect. B 43, 390 (1989).

[18] F. Sprenger, M. Lestinsky, D. A. Orlov, D. Schwalm, and A. Wolf, Nucl. Instrum. Methods Phys. Res., Sect. A 532, 298 (2004).

[19] S. Pastuszka, M. Hoppe, D. Kratzmann, D. Schwalm, A. Wolf, A. S. Jaroshevich, S. N. Kosolobov, D. A. Orlov, and A. S. Terekhov, J. Appl. Phys. 88, 6788 (2000).
[20] D. A. Orlov, F. Sprenger, M. Lestinsky, U. Weigel, A. S. Terekhov, D. Schwalm, and A. Wolf, J. Phys. Conf. Ser. 4, 290 (2005).

[21] C. Krantz, D. A. Orlov, J. Hoffmann, M. Lestinsky, O. Novotný, A. S. Jaroshevich, A. S. Terekhov, and A Wolf, J. Phys. Conf. Ser. 192, 012025 (2009).

[22] M. Hamberg, W. D. Geppert, R. D. Thomas, V.Zhaunerchyk, F. Österdahl, A. Ehlerding, M. Kaminska, J. Semaniak, M. af Ugglas, A. Källberg, A. Paal, A. Simonsson, and M. Larsson, Mol. Phys. 105, 899 (2007).

[23] M. Hamberg, F. Österdahl, R. D. Thomas, V. Zhaunerchyk, E. Vigren, M. Kaminska, M. af Ugglas, A. Källberg, A. Simonsson, A. Paál, M. Larsson, and W. D. Geppert, Astron. Astrophys. 514, A83 (2010).

[24] M. Hamberg, V. Zhaunerchyk, E. Vigren, M. Kaminska, I. Kashperka, M. Zhang, S. Trippel, F. Österdahl, M. af Ugglas, R. D. Thomas, A. Källberg, A. Simonsson, A. Paál, J. Semaniak, M. Larsson, and W. D. Geppert, Astron. Astrophys. 522, A90 (2010).

[25] R. von Hahn et al., Rev. Sci. Instrum. 87, 063115 (2016).

[26] H. Kreckel, O. Novotný, and A. Wolf, Phil. Trans. R. Soc. A 377, 20180412 (2019).

[27] D. A. Orlov, M. Lestinsky, F. Sprenger, D. Schwalm, A. S. Terekhov, and A. Wolf, AIP Conf. Proc. 821, 478 (2006).

[28] O. Novotný et al., Science 365, 676 (2019).

[29] R. Repnow, Pramana-J. Phys. 59, 835 (2002).

[30] M. Grieser et al., Eur. Phys. J. Special Topics 207, 1 (2012).

[31] M. Steck, G. Bisoffi, M. Blum, A. Friedrich, C. Geyer, M. Grieser, B. Holzer, E. Jaeschke, M. Jung, D. Krämer, K. Matl, W. Ott, and R. Repnow, Nucl. Instrum. Methods Phys. Res., Sect. A 287, 324 (1990).

[32] J. J. Scheer and J. van Laar, Solid State Commun. 3, 189 (1965).

[33] D. A. Orlov, U. Weigel, D. Schwalm, A. S. Terekhov, and A. Wolf, Nucl. Instrum. Methods Phys. Res., Sect. A 532, 418 (2004).

[34] D. A. Orlov, C. Krantz, A. Wolf, A. S. Jaroshevich, S. N. Kosolobov, H. E. Scheibler, and A. S. Terekhov, J. Appl. Phys. 106, 054907 (2009).

[35] M. Lestinsky, E. Lindroth, D. A. Orlov, E. W. Schmidt, S. Schippers, S. Böhm, C. Brandau, F. Sprenger, A. S. Terekhov, A. Müller, and A. Wolf, Phys. Rev. Lett. 100, 033001 (2008).

[36] D. A. Orlov, H. Fadil, M. Grieser, C. Krantz, J. Hoffmann, O. Novotný, S. Novotny, and A. Wolf, in Proc. of COOL 07International Workshop on Beam Cooling and Related Topics, editors R. W. Hasse and V. .R. W. Schaa (Gesellschaft für Schwerionenforschung mbH, Darmstadt, 2007), pp. 230234, https://accelconf.web.cern.ch/c107/papers/frm1c03.pdf.

[37] O. Novotný et al., J. Phys. Chem. A 114, 4870-4874 (2010).

[38] I. Nevo, S. Novotny, H. Buhr, V. Andrianarijaona, S. Altevogt, O. Heber, J. Hoffmann, H. Kreckel, L. Lammich, M. Lestinsky, H. B. Pedersen, D. Schwalm, A. Wolf, and D. Zajfman, Phys. Rev. A 76, 022713 (2007).

[39] D. Strasser, L. Lammich, H. Kreckel, M. Lange, S. Krohn, D. Schwalm, A. Wolf, and D. Zajfman, Phys. Rev. A 69 , 064702 (2004).

[40] H. Buhr, M. B. Mendes, O. Novotný, D. Schwalm, M. H. Berg, D. Bing, O. Heber, C. Krantz, D. A. Orlov, 
M. L. Rappaport, T. Sorg, J. Stützel, J. Varju, A. Wolf, and D. Zajfman, Phys. Rev. A 81, 062702 (2010).

[41] C. Krantz, K. Blaum, M. Grieser, Yu. A. Litvinov, R. Repnow, and A. Wolf, Nucl. Instrum. Methods Phys. Res., Sect. A 629, 1 (2011).

[42] M. Bell, J. Chaney, H. Herr, F. Krienen, P. Møller-Petersen, and G. Petrucci, Nucl. Instrum. Methods Phys. Res. 190, 237 (1981).

[43] M. Chanel, Nucl. Instrum. Methods Phys. Res., Sect. A 441, 64 (2000).

[44] A. Wolf, S. Krohn, H. Kreckel, L. Lammich, M. Lange, D. Strasser, M. Grieser, D. Schwalm, and D. Zajfman, Nucl. Instrum. Methods Phys. Res., Sect. A 532, 69 (2004).

[45] M. Larsson and A. E. Orel, Dissociative Recombination of Molecular Ions (Cambridge University Press, Cambridge, England, 2008).

[46] Ya. S. Derbenev and A. N. Skrinsky, Part. Accel. 8, 1 (1977).

[47] H. Danared, in Proc. of the 7th European Particle Accelerator Conference EPAC'00, Vienna, Austria (EPS, Geneva, 2000), pp. 1238-1240.

[48] Ya. S. Derbenev and A. N. Skrinsky, Part. Accel. 8, 235 (1978).

[49] V. V. Parkhomchuk and A. N. Skrinsky, Rep. Prog. Phys. 54, 919 (1991).

[50] S. Pastuszka, U. Schramm, M. Grieser, C. Broude, R. Grimm, D. Habs, J. Kenntner, H.-J. Miesner, T. Schüßler, D. Schwalm, and A. Wolf, Nucl. Instrum. Methods Phys. Res., Sect. A 369, 11 (1996).

[51] V. V. Parkhomchuk, Nucl. Instrum. Methods Phys. Res., Sect. A 441, 9 (2000).

[52] A. V. Fedotov, B. Gålnander, V. N. Litvinenko, T. Lofnes, A. Sidorin, A. Smirnov, and V. Ziemann, Phys. Rev. E 73, 066503 (2006).

[53] J. S. Bell and M. Bell, Part. Accel. 11, 233 (1981).
[54] N. S. Dikansky, V. I. Kudelainen, V. A. Lebedev, I. N. Meskov, V. V. Parkhomchuk, A. A. Sery, A. N. Skrinsky, and B. N. Sukhina, Ultimate possibilities of electron cooling, Institute of Nuclear Science, Novosibirsk, preprint No. 88-61, 1988.

[55] V. I. Kudelainen, V. A. Lebedev, I. N. Meshkov, V. V. Parkhomchuk, and B. N. Sukhina, Zh. Eksp. Teor. Fiz. 83, 2056-2064 (1982) [Sov. Phys. JETP 56, 1191 (1982)].

[56] H. Danared, Nucl. Instrum. Methods Phys. Res., Sect. A 391, 24 (1997).

[57] L. Spitzer, Jr., Physics of Fully Ionized Gases, 2nd ed. (Wiley \& Sons, New York, 1962), ch. 5.

[58] I. Langmuir, Phys. Rev. 2, 450 (1913).

[59] H. Danared, Phys. Scr. 48, 405 (1993).

[60] M. Martini, Report No. CERN PS/84-9 (AA).

[61] S. Artikova, M. Grieser, and J. Ullrich, in Proc. of the 2nd Intl. Particle Accelerator Conf. (IPAC'11), San Sebastián, Spain (EPS-AG, Spain, 2011), pp. 2490-2492.

[62] A. Shornikov, D. A. Orlov, C. Krantz, A. S. Jaroshevich, and A. Wolf, Phys. Rev. Accel. Beams 17, 042802 (2014).

[63] M. Beutelspacher, M. Grieser, D. Schwalm, and A. Wolf, Nucl. Instrum. Methods Phys. Res., Sect. A 441, 110 (2000).

[64] K. Hedblom and L. Hermansson, Nucl. Instrum. Methods Phys. Res., Sect. A 391, 37 (1997).

[65] H. Danared, G. Andler, L. Bagge, A. Källberg, F. Österdahl, A. Paál, M. Simonsson, and M. af Ugglas, in Proc. of the 7th European Particle Accelerator Conference EPAC'OO, Vienna, Austria (EPS, Geneva, 2000), pp. 301-303.

[66] M. Beutelspacher, M. Grieser, K. Noda, D. Schwalm, and A. Wolf, Nucl. Instrum. Methods Phys. Res., Sect. A 512, 459 (2003).

[67] B. Veglia, J. R. Hunt, J. Resta-Lopez, V. Rodin, and C. P. Welsch, in Proc. of the 9th Intl. Particle Accelerator Conf. (IPAC'18), Vancouver, Canada (JACoW, Geneva, 2018), pp. 2975-2978. 